\title{
A phylogenetically-based nomenclature for Cordycipitaceae (Hypocreales)
}

Ryan M. Kepler ${ }^{1}$, J. Jennifer Luangsa-ard ${ }^{2}$, Nigel L. Hywel-Jones ${ }^{3}$, C. Alisha Quandt ${ }^{4}$, Gi-Ho Sung ${ }^{5}$, Stephen A. Rehner ${ }^{6}$, M. Catherine Aime ${ }^{7}$, Terry W. Henkel ${ }^{8}$, Tatiana Sanjuan ${ }^{9}$, Rasoul Zare ${ }^{10}$, Mingjun Chen ${ }^{11}$, Zhengzhi Li ${ }^{3}$, Amy Y. Rossman ${ }^{12}$, Joseph W. Spatafora ${ }^{12}$, and Bhushan Shrestha ${ }^{13}$

1USDA-ARS, Sustainable Agriculture Systems Laboratory, Beltsville, MD 20705, USA; corresponding author e-mail: ryan.kepler@ars.usda.gov ${ }^{2}$ Microbe Interaction and Ecology Laboratory, BIOTEC, National Science and Technology Development Agency, 113 Thailand Science Park, Phahonyothin Rd, Klong Neung, Klong Luang, Pathum Thani, 12120 Thailand

${ }^{3}$ Zhejiang BioAsia Institute of Life Sciences, 1938 Xinqun Road, Economic and Technological Development Zone, Pinghu, Zhejiang, 314200 China

${ }^{4}$ Department of Ecology and Evolutionary Biology, University of Michigan, Ann Arbor, MI 48104, USA

5Institute for Bio-Medical Convergence, International St Mary's Hospital and College of Medicine, Catholic Kwandong University, Incheon 22711, Korea

${ }^{6}$ USDA-ARS, Mycology and Nematology Genetic Diversity and Biology Laboratory, Beltsville, MD 20705, USA

${ }^{7}$ Department of Botany and Plant Pathology, Purdue University, West Lafayette, IN 47907, USA

${ }^{8}$ Department of Biological Sciences, Humboldt State University, Arcata, CA, 95521, USA

'Laboratorio de Taxonomía y Ecología de Hongos, Universidad de Antioquia, calle 67 No. 53 - 108, A.A. 1226, Medellin, Colombia

${ }^{10}$ Iranian Research Institute of Plant Protection, Agricultural Research, Education and Extension Organization (AREEO), Tehran, Iran

${ }^{11}$ Anhui Provincial Key Laboratory of Microbial Control, Anhui Agricultural University, Hefei 230036, Peoples' Republic of China

${ }^{12}$ Department of Botany and Plant Pathology, Oregon State University, Corvallis, OR 97331, USA

${ }^{13}$ Mushtech Cordyceps Institute, Cheongil-ro 453 Beon-gil 55-9, Cheongil-myeon, Hoengseong-gun, Gangwon Province 25255, Korea

Abstract: The ending of dual nomenclatural systems for pleomorphic fungi in 2011 requires the reconciliation of competing names, ideally linked through culture based or molecular methods. The phylogenetic systematics of Hypocreales and its many genera have received extensive study in the last two decades, however resolution of competing names in Cordycipitaceae has not yet been addressed. Here we present a molecular phylogenetic investigation of Cordycipitaceae that enables identification of competing names in this family, and provides the basis upon which these names can be maintained or suppressed. The taxonomy presented here seeks to harmonize competing names by principles of priority, recognition of monophyletic groups, and the practical usage of affected taxa. In total, we propose maintaining nine generic names, Akanthomyces, Ascopolyporus, Beauveria, Cordyceps, Engyodontium, Gibellula, Hyperdermium, Parengyodontium, and Simplicillium and the rejection of eight generic names, Evlachovaea, Granulomanus, Isaria, Lecanicillium, Microhilum, Phytocordyceps, Synsterigmatocystis, and Torrubiella. Two new generic names, Hevansia and Blackwellomyces, and a new species, Beauveria blattidicola, are described. New combinations are also proposed in the genera Akanthomyces, Beauveria, Blackwellomyces, and Hevansia.
Key words: Akanthomyces Ascopolyporus Beauveria Blackwellomyces Cordyceps Dual nomenclature Engyodontium Gibellula Hevansia Hyperdermium Parengyodontium Simplicillium

Article info: Submitted: 21 April 2017; Accepted: 30 October 2017; Published: 21 November 2017.

\section{INTRODUCTION}

A molecular phylogenetic investigation of Clavicipitaceae, with an emphasis on Cordyceps, was conducted by Sung et al. (2007) and revealed that both Clavicipitaceae and Cordyceps were not monophyletic. Two additional families, Cordycipitaceae and Ophiocordycipitaceae, were recognized and species previously classified in Cordyceps were supported as members of all three families. Clavicipitaceae and Ophiocordycipitaceae collectively formed a monophyletic group, whereas Cordycipitaceae, defined by the phylogenetic position of the type species of Cordyceps, C. militaris, shared a more recent common ancestor with Hypocreaceae. The majority of sexually reproducing species in Cordyceps s. str. produce stalked, erect stromatic ascomata that are fleshy in texture, but some species are characterized by reduced stipes or subiculate stromata. Stromata are frequently bright yellow to orange or red, but others are pallid to cream or white according to species.

In addition to Cordyceps, numerous genera for sexual morphs have been included in Cordycipitaceae, the most speciose and taxonomically problematic being Torrubiella. Torrubiella has traditionally been used to classify pathogens of spiders or less frequently scale insects that produce superficial perithecia, frequently on a subiculum. Work by Johnson et al. (2009) showed the genus to be polyphyletic with species of Torrubiella being placed in Cordyceps and

\section{๑) 2017 International Mycological Association}

You are free to share - to copy, distribute and transmit the work, under the following conditions:

Attribution: $\quad$ You must attribute the work in the manner specified by the author or licensor (but not in any way that suggests that they endorse you or your use of the work).

Non-commercial: $\quad$ You may not use this work for commercial purposes.

No derivative works: You may not alter, transform, or build upon this work.

For any reuse or distribution, you must make clear to others the license terms of this work, which can be found at http://creativecommons.org/licenses/by-nc-nd/3.0/legalcode. Any of the above conditions can be waived if you get permission from the copyright holder. Nothing in this license impairs or restricts the author's moral rights. 
Ophiocordyceps (Ophiocordycipitaceae) as well as the new genera Conoideocrella and Orbiocrella of Clavicipitaceae. Torrubiella is now restricted to Cordycipitaceae infecting spiders with most possessing asexual morphs that had been referred to as Akanthomyces or Gibellula (Johnson et al. 2009, Evans 2013). Other genera in Cordycipitaceae produce perithecia on a subiculum, including Ascopolyporus and Hyperdermium, but these differ from Torrubiella in being pathogens of scale insects (Bischoff et al. 2005). Phytocordyceps is a monotypic genus described for $P$. ninchukispora, which molecular data determined to be nested within Cordyceps (Sung et al. 2007), although, it is unusual for the genus with respect to host affiliation and ascospore morphology. The host is reported as a seed of Beilschmiedia erythrophloia (Lauraceae), although closely related Cordyceps species attack pupae of Limacodidae, which superficially resemble globose seeds. The ascospores feature swollen ends connected by a long, narrowed midsection, a morphology referred to as bola-ascospores (Eriksson 1982) that are also present in C. bifusispora and C. cf. pruinosa (Sung et al. 2007).

Asexual morphs in Cordycipitaceae have been classified under many different names, and species of Cordyceps have been associated with a diversity of asexual reproductive morphologies. Many of these asexually typified genera have been demonstrated to be polyphyletic across Hypocreales. One of the oldest names for an asexually typified genus in Cordycipitaceae is Isaria. The use of Isaria has varied greatly over time and many associations outside of Hypocreales have been observed (Samson 1974, Luangsa-ard et al. 2004). The currently accepted concept of the genus was established by Hodge et al. (2005), who designated a drawing of $I$. farinosa by Holmskjold from 1781 as the lectotype for the genus. This definition set arthropod-infecting species in Hypocreales apart from morphologically similar Paecilomyces in Eurotiales. Molecular data supported this distinction (Luangsa-ard et al. 2004), but also revealed a polyphyletic distribution of Isaria species in Hypocreales (Luangsa-ard et al. 2005). Taxonomic transfers for species with isarioid morphologies in the families Clavicipitaceae and Ophiocordycipitaceae have also been made in more inclusive investigations (Johnson et al. 2009, Luangsa-ard et al. 2011, Kepler et al. 2014, Quandt et al. 2014, Ban et al. 2015, Spatafora et al. 2015).

Within Cordycipitaceae, the asexually typified generic names Lecanicillium and Simplicillium were described for verticillium-like taxa. Species in Lecanicillium are distributed throughout Cordycipitaceae and do not form a single monophyletic clade (Sukarno et al. 2009). The type of Lecanicillium is $L$. lecanii, known as the asexual morph of $C$. confragosa, which was described as a Torrubiella based on its host association with scale insects and the production of superficial torrubielloid perithecia (Evans \& Samson 1982). The asexual morph of $C$. militaris is also morphologically similar to Lecanicillium (Gams \& Zare 2001). Relationships for species of Lecanicillium include affinities with isolates morphologically similar to $I$. farinosa and a unique clade of species characterized by L. psalliotae (Sung et al. 2001, 2007). Simplicillium identifies a clade of species that is sister to the remaining Cordycipitaceae; no known sexual form has been linked to the genus (Nonaka et al. 2013).
The morphology is that of slender, elongate, mostly solitary phialides producing singular or short chains of conidia (Zare $\&$ Gams 2001). These species are mostly pathogens of fungi, although occurrence on other substrates (e.g. air, soil, plants) is known (Nonaka et al. 2013).

Perhaps the best-known asexually typified generic name in Cordycipitaceae is Beauveria, due to its role in the development of the germ theory of disease and its long-standing use as a biocontrol agent against pest insects. Agostino Bassi demonstrated in 1834 that $B$. bassiana (a fungus that bears his name) was the cause of white muscardine disease of silkworm, which resulted in considerable loss to the silk industry in Europe (Yarrow 1958). This represented the first demonstration of pathogenic activity by a disease agent, predating Pasteur, who cited Bassi in his papers (Porter 1973). Today, the pathogenic ability of $B$. bassiana is intensely scrutinized in the effort to improve control of insect pests (Xiao et al. 2012). Beauveria is defined by the distinctive conidiogenous cells that elongate in a sympodial pattern to produce a zig-zag rachis-like structure. Molecular phylogenies support the monophyly of Beauveria, confirming the phylogenetic utility of this form of conidiogenesis, especially when combined with other characters (e.g., hydrophobic conidia, insect pathogenicity; Rehner et al. 2011). The genus is highly diverse, with many cryptic species, and links to several sexually typified species in Cordyceps (Rehner \& Buckley 2005, Rehner et al. 2011). These include the moth pathogen C. bassiana (Huang et al. 2002), and the beetle pathogens $C$. brongniartii (Shimazu et al. 1988) and C. scarabaeicola (Shrestha et al. 2014a), as well as pathogens of grasshoppers, stick insects, and cockroaches from South America (Sanjuan et al. 2014), and crickets from Thailand (Ariyawansa et al. 2015).

Other asexually typified genera that produce conidia on a rachis-like conidiophore include Evlachovaea, Engyodontium, and Parengyodontium. A molecular investigation of Evlachovaea based on ITS and TEF data, including material from the ex-type strain of E. kintrischica, revealed a close association with some species placed in Isaria (Humber et al. 2013). They demonstrated this generic name was a later synonym of Isaria, but no formal transfer to the genus was made at that time. Likewise, molecular data have supported the separation of Engyodontium from Beauveria, and the classification of $P$. album as distinct from Engyodontium (Tsang et al. 2016). Finally, the monotypic genus Microhilum was described for the asexual morph of a Cordyceps (Yip \& Rath 1989). It produces short conidiophores that give rise to conidium-producing denticles and is morphologically similar to Beauveria and Isaria; molecular data, however, place this species close to C. militaris and C. kyusyuensis (Sung et al. 2007).

Several asexually typified genera are associated with Torrubiella, the two most common being Akanthomyces and Gibellula. A revision of Akanthomyces by Mains (1950) included species forming hyaline conidia of various shapes on phialides covering a cylindrical synnema in a hymenium-like layer, with superficial similarity to species of Hymenostilbe. The type species of Akanthomyces, A. aculeatus, primarily infects Lepidoptera. Cordyceps tuberculata is linked to $A$. pistillariiformis, a pathogen of moths and a close relative of 
A. aculeatus. Gibellula species are pathogens of spiders and produce synnemata with swollen conidiophores reminiscent of Aspergillus. Other minor asexual genera include Granulomanus, which is now considered to be a synonym of Gibellula (Humber \& Rombach 1987), and Pseudogibellula, which is a synonym of Ophiocordyceps (Spatafora et al. 2015).

As summarized above, the taxonomic history of Cordycipitaceae is complex and involves numerous sexual and asexually typified names that have been used throughout Hypocreales. Here we present a phylogenetically informed resolution of competing generic names in Cordycipitaceae in order to determine the generic names to use since the end of dual nomenclature for different morphs of the same fungus in 2011 (McNeill et al. 2012). In making decisions on names, we sought to harmonize the competing interests among name priority, preferences of user communities, the number of name changes required, and recognition of monophyletic groups from molecular phylogenetic analyses. We also introduce new generic and species names where data support a straightforward taxonomic solution.

\section{MATERIALS AND METHODS}

This work employs the data used in name reconciliation for Ophiocordycipitaceae by Quandt et al. (2014), additional published sequences obtained from GenBank (Bischoff et al. 2005, Rehner et al. 2011, Humber et al. 2013, Sanjuan et al. 2014), and sequences determined as part of this work (Table 1). Phylogenetic analyses utilized data from five nuclear genes, including the small and large subunits of nuclear encoded ribosomal DNA genes (SSU and LSU), the protein coding genes translation elongation factor 1 alpha (TEF), and the largest (RPB1) and second largest (RPB2) subunits of RNA polymerase II, and representatives for the type species of sexual and asexual genera throughout the family (Table 1). After assembly of raw sequencing reads with CodonCode Aligner, version 2.0.6 (Dedham, MA) sequences were aligned with representative sequences from throughout Hypocreales as in Quandt et al. (2014) using MAFFT v.6 (Katoh et al. 2002, Katoh \& Toh 2008). After alignment, gaps, introns and ambiguously aligned regions identified with Gblocks (Talavera \& Castresana 2007) were removed using the editing capacity of Geneious v. 7.0.6 (Biomatters; available from http://www. geneious.com). Maximum likelihood analysis was performed with RAXML v. 8.2.8 employing a GTRGAMMA model of nucleotide substitution. The dataset was divided into eleven separate partitions, one for each ribosomal gene and one each for of the three codon positions in protein coding genes. The resulting phylogenetic framework serves as a guide to resolve conflicts between competing names for sexually and asexually typified generic names.

For description of new species, collections were rehydrated in sterilized water. Perithecia, asci, ascospores and part-spores were examined on a Leica DMRB compound microscope and Leica M28 stereomicroscope. Methuen Handbook of Color (Kornerup et al. 1984) was used for colour descriptions of stromata.

\section{RESULTS}

The overall topology recovered in this analysis agrees with that of previous works (Fig. 1; Sung et al. 2007, Johnson et al. 2009, Nonaka et al. 2013, Quandt et al. 2014). The family Cordycipitaceae is well supported, as are many of the internal nodes, and this phylogenetic hypothesis for the family supports the recognition of the genera Akanthomyces, Ascopolyporus, Beauveria, Cordyceps, Engyodontium, Gibellula, Hyperdermium, and Simplicillium. Parengyodontium was not sampled due to insufficient sequence data. Additionally, we describe two new genera, Hevansia and Blackwellomyces, to accommodate two clades of species without available generic names, and a new species of Beauveria, B. blattidicola, that infects cockroaches. We do not use Evlachovaea, Isaria, Lecanicillium, Microhilum, and Torrubiella and propose that they be rejected along with other lesser-known names (Table 2). There exist clades and lineages containing species named in Cordyceps, Lecanicillium and Torrubiella that are not members of the clades containing the type species of those genera; these are effectively treated as incertae sedis. Below we discuss the major genera of Cordycipitaceae for which sufficient sampling is available. In all cases, proposed genera are the least inclusive clades defined in the reference phylogeny as the terminal generic clade (Fig. 1).

\section{TAXONOMY}

Akanthomyces Lebert, Z. Wiss. Zool. 9: 449 (1858).

Type: Akanthomyces aculeatus Lebert, Z. Wiss. Zool. 9: 449 (1858).

The genus Akanthomyces as proposed by Lebert (1858), including the type $A$. aculeatus, primarily infects Lepidoptera and forms a clade distinct from Beauveria and Cordyceps. It includes the moth pathogen Cordyceps tuberculata, which is linked to an asexual morph described as $A$. pistillariiformis (Samson \& Evans 1974). Other fungi in this clade include C. coccidioperitheciata and C. confragosa, pathogens of spiders and scales insects, respectively, which produce torrubielloid perithecia (Kobayasi \& Shimizu 1982). The production of superficial perithecia on a stipe distinguishes C. coccidioperitheciata from other sexual forms infecting spiders in Cordycipitaceae, which either lack a stipe or lack superficial perithecia if a stipe is present. Cordyceps confragosa was described by Mains (1949) in Torrubiella, and while the morphology is torrubielloid, Akanthomyces has taxonomic priority by date over Torrubiella (Boudier 1885). In addition, the sexual morph $C$. confragosa is linked to Lecanicillium lecanii, the type species of Lecanicillium, now considered a synonym of Akanthomyces, which has priority over Lecanicillium (Gams \& Zare 2001). Chiriví-Salomón et al. (2015) also showed that L. lecanii (as C. confragosa) as well as some other species of Lecanicillium, namely $L$. attenuatum, L. muscarium, and L. sabanense, fall within Akanthomyces. Akanthomyces also includes asexually typified species names previously assigned to the genus Isaria, but not the type species, I. farinosa, which belongs 
Table 1. Proposed list of generic names in Cordycipitaceae to be protected and their competing synonyms.

Proposed to protect

Akanthomyces Lebert in Z. Wiss. Zool. 9: 449. 1858.

Typus: Akanthomyces aculeatus Lebert 1858.
Proposed to reject

(=) Torrubiella Boud. in Rev Mycol. (Toulouse) 7: 226. 1885 Typus: T. aranicida Boud. 1885

(=) Lecanicillium W. Gams \& Zare in Nova Hedwigia 72: 50. 2001.

Typus: L. lecanii (Zimm.) Zare \& W. Gams 2001, now regarded as Akanthomyces lecanii (Zimm.) Spatafora et al. 2017

Ascopolyporus Möller in Bot. Mitt. Tropen 9: 300. 1901.

Typus: Ascopolyporus polychrous Möller 1901.

Beauveria Vuill. in Bull. Soc. Bot. France. 59: 40. 1912.

Typus: Beauveria bassiana (Bals.-Criv.) Vuill. 1912 (Botrytis bassiana Bals.Criv. 1835).

Blackwellomyces Spatafora \& Luangsa-ard in IMA Fungus 8: 345. 2017.

Typus: Blackwelliella cardinalis (G.H. Sung \& Spatafora) Spatafora \& Luangsaard 2017) Cordyceps cardinalis G.H. Sung \& Spatafora 2004).

Cordyceps Fr., Observ. Mycol. 2: 316 [cancellans)] 1818, nom. cons.

Typus: Cordyceps militaris (L.) Fr. 1818 (Clavaria militaris L.1753).

(=) Isaria Pers. in Neues Mag.Bot.1: 121. 1794.

Typus: I. farinosa (Holmsk.) Fr. 1832, now regarded as Cordyceps farinosa (Holmsk.) Kepler et al. 2017.

(=) Microhilum H.Y. Yip \& A.C. Rath in J. Invert. Path. 53: 361. 1989.

Typus: M. oncoperae H.Y. Yip \& A.C. Rath 1989, now regarded as Cordyceps oncoperae (H.Y. Yip \& A.C. Rath) Kepler et al. 2017.

(=) Phytocordyceps C.H. Su \& H.H. Wang in Mycotaxon 26: 338. 1986.

Typus: P. ninchukispora C.H. Su \& H.H. Wang 1986. now regarded as Cordyceps ninchukispora (C.H. Su \& H.H. Wang) G.H. Sung et al. 2007.

(=) Evlachovaea Borisov \& Tarasov in Mikol. Fitopatol. 33: 250. 1999.

Typus: E. kintrischica B.A. Borisov \& Tarasov 1999, now regarded as Cordyceps kintrischica (B.A. Borisov \& Tarasov) Kepler et al. 2017.

Engyodontium de Hoog in Persoonia 10: 53. 1978.

Typus: Engyodontium parvisporum (Petch) de Hoog 1978 (Rhinotrichum parvispora Petch 1932).

Gibellula Cavara in Atti Ist. Bot. R. Univ. Pavia, ser. 2 3: 347. 1894.

Typus: Gibellula pulchra Cavara 1894.

(=) Synsterigmatocystis Costantin in Bull. Soc. Mycol. France 4: 63. 1888

Typus: S. arachnophila Costantin ex Vuill. 1888, now regarded as Gibellula arachnophila (Ditmar) Vuill. 1910.

(=) Granulomanus de Hoog \& Samson in Persoonia 10: 70. 1978.

Typus: G. aranearum (Petch) de Hoog \& Samson 1978, basionym: Cylindrophora aranearum Petch 1944.

Hevansia Luangsa-ard, Hywel-Jones \& Spatafora in IMA Fungus 8: 348. 2017.

Typus: Hevansia novoguineensis (Samson \& B.L. Brady) Luangsa-ard, HywelJones \& Spatafora 2017 (Akanthomyces novoguineensis Samson \& B.L. Brady 1982).

Hyperdermium J.F. White et al. in Mycologia 92: 910. 2000.

Typus: Hyperdermium caulium (Berk. \& M.A. Curtis) Chaverri \& K.T. Hodge 2008 (Corticium caulium Berk. \& M.A. Curtis 1854 [1853]).

Parengyodontium C.C. Tsang et al. in Medical Mycology 54: 708. 2016.

Typus: Parengyodontium album (Limber) C.C. Tsang et al. 2016 (Tritirachium album Limber 1940).

Simplicillium W. Gams \& Zare in Nova Hedwigia 73: 38, 2001.

Typus: Simplicillium lanosiniveum (J.F.H. Beyma) Zare \& W. Gams 2001

(Phalosporium lanosoniveum J.F.H. Beyma 1942). 


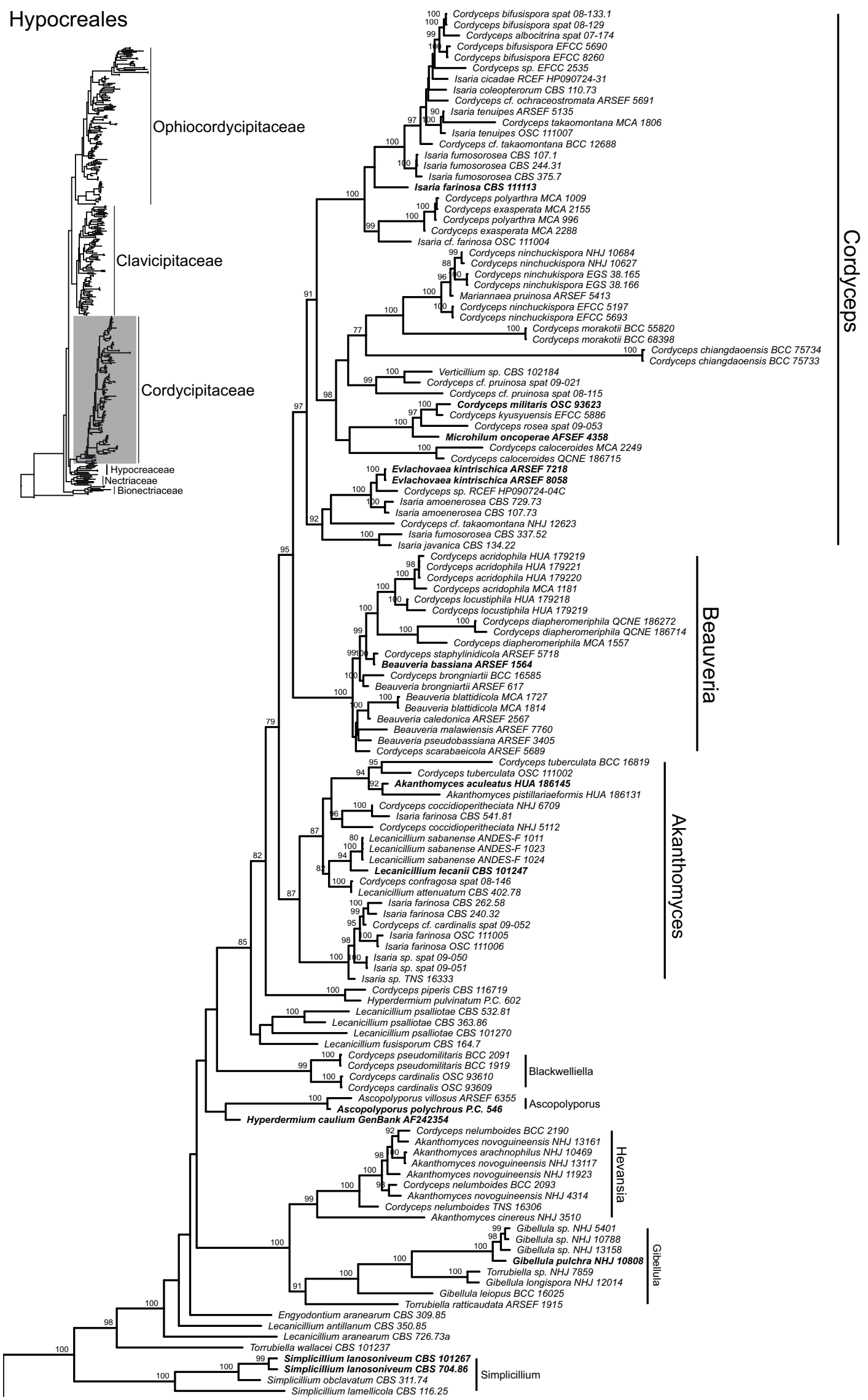

Fig. 1. RAXML tree of Cordycipitaceae. Dataset included 392 taxa and a concatenated alignment of 4569 bp from five nuclear genes (SSU, LSU, TEF, RPB1, and RPB2). Tips in bold represent examples of type species for their associated genera. Proposed genus level names to protect are delimited, but names of individual species have not been changed on the leaves of the tree, demonstrating diversity of taxa sampled. Values above branches are bootstrap proportions. 
Table 2. Voucher information and Genbank numbers for samples appearing in Figure 1.

\begin{tabular}{|c|c|c|c|c|c|c|}
\hline Species & Collection & nrSSU & nrLSU & TEF & RPB1 & RPB2 \\
\hline Akanthomyces aculeatus & HUA 186145 & MF416572 & MF416520 & MF416465 & & \\
\hline $\begin{array}{l}\text { Akanthomyces } \\
\text { arachnophilus }\end{array}$ & NHJ 10469 & EU369090 & EU369031 & EU369008 & EU369047 & \\
\hline Akanthomyces cinereus & NHJ 3510 & EU369091 & & EU369009 & EU369048 & EU369070 \\
\hline $\begin{array}{l}\text { Akanthomyces } \\
\text { novoguineensis }\end{array}$ & NHJ 13117 & EU369092 & & EU369010 & EU369049 & EU369073 \\
\hline $\begin{array}{l}\text { Akanthomyces } \\
\text { novoguineensis }\end{array}$ & NHJ 13161 & EU369093 & & EU369011 & EU369050 & \\
\hline $\begin{array}{l}\text { Akanthomyces } \\
\text { novoguineensis }\end{array}$ & NHJ 4314 & EU369094 & & EU369012 & EU369051 & EU369071 \\
\hline $\begin{array}{l}\text { Akanthomyces } \\
\text { novoguineensis }\end{array}$ & NHJ 11923 & EU369095 & EU369032 & EU369013 & EU369052 & EU369072 \\
\hline $\begin{array}{l}\text { Akanthomyces } \\
\text { pistillariaeformis }\end{array}$ & HUA 186131 & MF416573 & MF416521 & MF416466 & & \\
\hline Ascopolyporus polychrous & P.C. 546 & & DQ118737 & DQ118745 & DQ127236 & \\
\hline Ascopolyporus villosus & ARSEF 6355 & & AY886544 & DQ118750 & DQ127241 & \\
\hline Beauveria bassiana & ARSEF 1564 & & & HQ880974 & HQ880833 & HQ880905 \\
\hline Beauveria blattidicola & MCA 1727 & MF416593 & MF416539 & MF416483 & MF416640 & \\
\hline Beauveria blattidicola & MCA 1814 & MF416594 & MF416540 & MF416484 & MF416641 & \\
\hline Beauveria brongniartii & ARSEF 617 & & & HQ880991 & HQ880854 & HQ880926 \\
\hline Beauveria caledonica & ARSEF 2567 & AF339570 & AF339520 & EF469057 & EF469086 & \\
\hline Beauveria malawiensis & ARSEF 7760 & & & DQ376246 & HQ880897 & HQ880969 \\
\hline Beauveria pseudobassiana & ARSEF 3405 & & & AY531931 & HQ880864 & HQ880936 \\
\hline Cordyceps acridophila & MCA 1181 & MF416574 & MF416522 & & MF416628 & \\
\hline Cordyceps acridophila & HUA 179220 & JQ895527 & JQ895536 & JQ958614 & JX003852 & JX003842 \\
\hline Cordyceps acridophila & HUA 179219 & & JQ895541 & JQ958613 & JX003857 & JX003841 \\
\hline Cordyceps acridophila & HUA 179221 & JQ895526 & JQ895537 & JQ958615 & JX003853 & JX003843 \\
\hline Cordyceps albocitrina & spat $07-174$ & MF416575 & & MF416467 & MF416629 & \\
\hline Cordyceps bifusispora & EFCC 5690 & EF468952 & EF468806 & EF468746 & EF468854 & EF468909 \\
\hline Cordyceps bifusispora & EFCC 8260 & EF468953 & EF468807 & EF468747 & EF468855 & EF468910 \\
\hline Cordyceps bifusispora & spat $08-129$ & MF416576 & MF416523 & MF416468 & MF416630 & \\
\hline Cordyceps bifusispora & spat $08-133.1$ & MF416577 & MF416524 & MF416469 & MF416631 & MF416434 \\
\hline Cordyceps brongniartii & BCC 16585 & JF415951 & JF415967 & JF416009 & JN049885 & JF415991 \\
\hline Cordyceps caloceroides & MCA 2249 & MF416578 & MF416525 & MF416470 & MF416632 & \\
\hline Cordyceps caloceroides & QCNE 186715 & MF416579 & MF416526 & & & \\
\hline Cordyceps cardinalis & OSC 93609 & AY184973 & AY184962 & DQ522325 & DQ522370 & DQ522422 \\
\hline Cordyceps cardinalis & OSC 93610 & AY184974 & AY184963 & EF469059 & EF469088 & EF469106 \\
\hline Cordyceps cf. cardinalis & spat 09-052 & MF416580 & MF416527 & MF416471 & MF416633 & MF416435 \\
\hline $\begin{array}{l}\text { Cordyceps cf. } \\
\quad \text { ochraceostromata }\end{array}$ & ARSEF 5691 & EF468964 & EF468819 & EF468759 & EF468867 & EF468921 \\
\hline Cordyceps cf. pruniosa & spat $08-115$ & MF416586 & MF416532 & MF416476 & MF416635 & MF416439 \\
\hline Cordyceps cf. pruniosa & spat 09-021 & MF416587 & MF416533 & MF416477 & MF416636 & \\
\hline $\begin{array}{l}\text { Cordyceps cf. } \\
\text { takaomontana }\end{array}$ & NHJ 12623 & EF468984 & EF468838 & EF468778 & EF468884 & EF468932 \\
\hline $\begin{array}{l}\text { Cordyceps cf. } \\
\text { takaomontana }\end{array}$ & BCC 12688 & MF416599 & MF416545 & MF416489 & MF416646 & \\
\hline $\begin{array}{l}\text { Cordyceps } \\
\quad \text { coccidioperitheciata }\end{array}$ & NHJ 5112 & EU369109 & EU369043 & EU369026 & EU369066 & \\
\hline $\begin{array}{l}\text { Cordyceps } \\
\quad \text { coccidioperitheciata }\end{array}$ & NHJ 6709 & EU369110 & EU369042 & EU369025 & EU369067 & EU369086 \\
\hline Cordyceps confragosa & spat 08-146 & MF416581 & MF416528 & MF416472 & MF416634 & MF416436 \\
\hline $\begin{array}{l}\text { Cordyceps } \\
\quad \text { diapheromeriphila }\end{array}$ & MCA 1557 & MF416582 & MF416529 & & & \\
\hline
\end{tabular}


Table 2. (Continued).

\begin{tabular}{|c|c|c|c|c|c|c|}
\hline Species & Collection & nrSSU & nrLSU & TEF & RPB1 & RPB2 \\
\hline $\begin{array}{l}\text { Cordyceps } \\
\quad \text { diapheromeriphila }\end{array}$ & QCNE 186714 & MF416601 & MF416547 & MF416491 & MF416648 & \\
\hline $\begin{array}{l}\text { Cordyceps } \\
\quad \text { diapheromeriphila }\end{array}$ & QCNE 186272 & JQ895530 & JQ895534 & JQ958610 & JX003848 & \\
\hline Cordyceps exasperata & MCA 2155 & MF416596 & MF416542 & MF416486 & MF416643 & \\
\hline Cordyceps exasperata & MCA 2288 & MF416592 & MF416538 & MF416482 & MF416639 & \\
\hline Cordyceps kyusyuensis & EFCC 5886 & EF468960 & EF468813 & EF468754 & EF468863 & EF468917 \\
\hline Cordyceps locustiphila & HUA 179218 & JQ895525 & JQ895535 & JQ958619 & JX003846 & JX003845 \\
\hline Cordyceps locustiphila & HUA 179219 & JQ958598 & JQ958597 & & JX003847 & \\
\hline Cordyceps militaris & OSC 93623 & AY184977 & AY184966 & DQ522332 & DQ522377 & AY545732 \\
\hline Cordyceps nelumboides & BCC 2093 & MF416583 & MF416530 & MF416473 & & MF416437 \\
\hline Cordyceps nelumboides & BCC 2190 & MF416584 & MF416531 & MF416474 & & \\
\hline Cordyceps nelumboides & TNS 16306 & MF416585 & & MF416475 & & MF416438 \\
\hline Cordyceps ninchuckispora & EFCC 5197 & EF468965 & EF468820 & EF468760 & EF468868 & \\
\hline Cordyceps ninchuckispora & EFCC 5693 & EF468966 & EF468821 & EF468762 & EF468869 & \\
\hline Cordyceps ninchuckispora & NHJ 10627 & EF468967 & IEF468822 & EF468763 & EF468870 & \\
\hline Cordyceps ninchuckispora & NHJ 10684 & EF468968 & EF468823 & EF468761 & EF468871 & \\
\hline Cordyceps ninchukispora & EGS 38.165 & EF468991 & EF468846 & EF468795 & EF468900 & \\
\hline Cordyceps ninchukispora & EGS 38.166 & EF468992 & EF468847 & EF468794 & EF468901 & \\
\hline Cordyceps piperis & CBS 116719 & & AY466442 & DQ118749 & DQ127240 & EU369083 \\
\hline Cordyceps polyarthra & MCA 996 & MF416597 & MF416543 & MF416487 & MF416644 & \\
\hline Cordyceps polyarthra & MCA 1009 & MF416598 & MF416544 & MF416488 & MF416645 & \\
\hline Cordyceps pseudomilitaris & BCC 1919 & MF416588 & MF416534 & MF416478 & & MF416440 \\
\hline Cordyceps pseudomilitaris & BCC 2091 & MF416589 & MF416535 & MF416479 & & MF416441 \\
\hline Cordyceps rosea & spat 09-053 & MF416590 & MF416536 & MF416480 & MF416637 & MF416442 \\
\hline Cordyceps scarabaeicola & ARSEF 5689 & AF339574 & AF339524 & DQ522335 & DQ522380 & DQ522431 \\
\hline Cordyceps sp. & EFCC 2535 & EF468980 & EF468835 & EF468772 & & \\
\hline Cordyceps sp. & $\begin{array}{l}\text { RCEF } \\
\text { HP090724-04C }\end{array}$ & MF416591 & MF416537 & MF416481 & MF416638 & MF416443 \\
\hline Cordyceps staphylinidicola & ARSEF 5718 & EF468981 & EF468836 & EF468776 & EF468881 & \\
\hline Cordyceps takaomontana & MCA 1806 & MF416595 & MF416541 & MF416485 & MF416642 & \\
\hline Cordyceps tuberculata & OSC 111002 & DQ522553 & DQ518767 & DQ522338 & DQ522384 & DQ522435 \\
\hline Cordyceps tuberculata & BCC 16819 & MF416600 & MF416546 & MF416490 & MF416647 & MF416444 \\
\hline Engyodontium aranearum & CBS 309.85 & AF339576 & AF339526 & DQ522341 & DQ522387 & DQ522439 \\
\hline Evlachovaea kintrischica & ARSEF 7218 & & & GU734751 & & \\
\hline Evlachovaea kintrischica & ARSEF 8058 & & & GU734750 & & \\
\hline Gibellula leiopus & BCC 16025 & MF416602 & MF416548 & MF416492 & MF416649 & \\
\hline Gibellula longispora & NHJ 12014 & EU369098 & & EU369017 & EU369055 & EU369075 \\
\hline Gibellula pulchra & NHJ 10808 & EU369099 & EU369035 & EU369018 & EU369056 & EU369076 \\
\hline Gibellula sp. & NHJ 10788 & EU369101 & EU369036 & EU369019 & EU369058 & EU369078 \\
\hline Gibellula sp. & NHJ 13158 & EU369100 & EU369037 & EU369020 & EU369057 & EU369077 \\
\hline Gibellula sp. & NHJ 5401 & EU369102 & & & EU369059 & EU369079 \\
\hline Hyperdermium caulium & $\begin{array}{l}\text { GenBank } \\
\text { AF242354 }\end{array}$ & & AF242354 & & & \\
\hline Hyperdermium pulvinatum & P.C. 602 & & DQ118738 & DQ118746 & DQ127237 & \\
\hline Isaria amoenerosea & CBS 107.73 & AY526464 & MF416550 & MF416494 & MF416651 & MF416445 \\
\hline Isaria amoenerosea & CBS 729.73 & MF416604 & MF416551 & MF416495 & MF416652 & MF416446 \\
\hline Isaria cf. farinosa & OSC 111004 & EF468986 & EF468840 & EF468780 & EF468886 & \\
\hline Isaria cicadae & $\begin{array}{l}\text { RCEF } \\
\text { HP090724-31 }\end{array}$ & MF416605 & MF416552 & MF416496 & MF416653 & MF416447 \\
\hline
\end{tabular}


Table 2. (Continued)

\begin{tabular}{|c|c|c|c|c|c|c|}
\hline Species & Collection & nrSSU & nrLSU & TEF & RPB1 & RPB2 \\
\hline Isaria coleopterorum & CBS 110.73 & JF415965 & JF415988 & JF416028 & JN049903 & JF416006 \\
\hline Isaria farinosa & OSC 111005 & DQ522558 & DQ518772 & DQ522348 & DQ522394 & \\
\hline Isaria farinosa & OSC 111006 & EF469127 & EF469080 & EF469065 & EF469094 & \\
\hline Isaria farinosa & CBS 240.32 & JF415958 & JF415979 & JF416019 & JN049895 & JF415999 \\
\hline Isaria farinosa & CBS 262.58 & AB023943 & AB080087 & MF416497 & MF416654 & MF416448 \\
\hline Isaria farinosa & CBS 541.81 & MF416606 & MF416553 & MF416498 & MF416655 & MF416449 \\
\hline Isaria farinosa & CBS 111113 & AY526474 & MF416554 & MF416499 & MF416656 & MF416450 \\
\hline Isaria fumosorosea & CBS 337.52 & MF416607 & MF416555 & MF416500 & MF416657 & MF416451 \\
\hline Isaria fumosorosea & CBS 375.70 & AB083035 & AB083035 & MF416501 & MF416658 & MF416452 \\
\hline Isaria fumosorosea & CBS 107.10 & MF416608 & MF416556 & MF416502 & MF416659 & MF416453 \\
\hline Isaria fumosorosea & CBS 244.31 & MF416609 & MF416557 & MF416503 & MF416660 & MF416454 \\
\hline Isaria javanica & CBS 134.22 & MF416610 & MF416558 & MF416504 & MF416661 & MF416455 \\
\hline Isaria sp. & TNS 16333 & MF416611 & & MF416505 & MF416662 & MF416456 \\
\hline Isaria sp. & spat $09-050$ & MF416613 & MF416559 & MF416506 & MF416663 & MF416457 \\
\hline Isaria sp. & spat $09-051$ & MF416614 & MF416560 & MF416507 & MF416664 & MF416458 \\
\hline Isaria tenuipes & OSC 111007 & DQ522559 & DQ518773 & DQ522349 & DQ522395 & DQ522449 \\
\hline Isaria tenuipes & ARSEF 5135 & MF416612 & JF415980 & JF416020 & JN049896 & JF416000 \\
\hline Lecanicillium antillanum & CBS 350.85 & AF339585 & AF339536 & DQ522350 & DQ522396 & DQ522450 \\
\hline Lecanicillium aranearum & CBS $726.73 a$ & AF339586 & AF339537 & EF468781 & EF468887 & EF468934 \\
\hline Lecanicillium attenuatum & CBS 402.78 & AF339614 & AF339565 & EF468782 & EF468888 & EF468935 \\
\hline Lecanicillium fusisporum & CBS 164.7 & AF339598 & AF339549 & EF468783 & EF468889 & \\
\hline Lecanicillium lecanii & CBS 101247 & AF339604 & AF339555 & DQ522359 & DQ522407 & DQ522466 \\
\hline Lecanicillium psalliotae & CBS 532.81 & AF339609 & AF339560 & EF469067 & EF469096 & EF469112 \\
\hline Lecanicillium psalliotae & CBS 101270 & EF469128 & EF469081 & EF469066 & EF469095 & EF469113 \\
\hline Lecanicillium psalliotae & CBS 363.86 & AF339608 & AF339559 & EF468784 & EF468890 & \\
\hline Mariannaea pruinosa & ARSEF 5413 & AY184979 & AY184968 & DQ522351 & DQ522397 & DQ522451 \\
\hline Microhilum oncoperae & AFSEF 4358 & AF339581 & AF339532 & EF468785 & EF468891 & EF468936 \\
\hline Simplicillium lamellicola & CBS 116.25 & AF339601 & AF339552 & DQ522356 & DQ522404 & DQ522462 \\
\hline Simplicillium lanosoniveum & CBS 101267 & AF339603 & AF339554 & DQ522357 & DQ522405 & DQ522463 \\
\hline Simplicillium lanosoniveum & CBS 704.86 & AF339602 & AF339553 & DQ522358 & DQ522406 & DQ522464 \\
\hline Simplicillium obclavatum & CBS 311.74 & AF339567 & AF339517 & EF468798 & & \\
\hline Torrubiella ratticaudata & ARSEF 1915 & DQ522562 & DQ518777 & DQ522360 & DQ522408 & DQ522467 \\
\hline Torrubiella sp. & NHJ 7859 & EU369107 & & & EU369064 & EU369085 \\
\hline Torrubiella wallacei & CBS 101237 & AY184978 & AY184967 & EF469073 & EF469102 & EF469119 \\
\hline Verticillium sp. & CBS 102184 & AF339613 & AF339564 & EF468803 & EF468907 & EF468948 \\
\hline
\end{tabular}

in Cordyceps. In general, the host range for asexual and sexual forms of Akanthomyces are similar, although $L$. attenuatum (CBS 402.78) was cultured from leaf litter with no host reported. The morphological characters associated with Akanthomyces are also found in a clade of spider-pathogenic species sister to the Gibellula clade (see Hevansia below).

The type species of Torrubiella, T. aranicida, known from a spider in France, was not available for inclusion in molecular phylogenetic analyses. However, several morphological characteristics of $T$. aranicida suggest that it may belong in Akanthomyces. These include the superficial and separated arrangement of the perithecia and the lack of a subiculum in the type specimen as shown in Johnson et al. (2009). A number of sexual morphs now placed in Akanthomyces have torrubielloid ascomata, specifically $A$. coccidioperitheciatus on spiders, A. lecanii on scale insects, and A. tuberculata on moths.

Akanthomyces attenuatus (Zare \& W. Gams) Spatafora, Kepler \& B. Shrestha, comb. nov.

MycoBank MB820860

Basionym: Lecanicillium attenuatum Zare \& W. Gams, Nova Hedwigia 73: 19 (2001).

Akanthomyces coccidioperitheciatus (Kobayasi \& Shimizu) Spatafora, Kepler \& B. Shrestha, comb. nov.

MycoBank MB820880

Basionym: Cordyceps coccidioperitheciata Kobayasi \& Shimizu, Bull. Natl. Sci. Mus. Tokyo, B 8: 79 (1982). 
Akanthomyces dipterigenus (Petch) Spatafora, Kepler, Zare \& B. Shrestha, comb. nov.

MycoBank MB823235

Basionym: Cephalosporium dipterigenum Petch, Naturalist (Hull) 56: 102 (1931).

Synonyms: Cephalosporium longisporum Petch, Trans. Brit. Mycol. Soc. 10: 166 (1925).

Lecanicillium longisporum (Petch) Zare \& W. Gams, Nova Hedwigia 73: 16 (2001).

? Acrostalagmus aphidum Oudem., Nederl. Kruidk. Arch. 3(2): 759 (1902) [no type collection found in L, only a drawing which does not allow a definite conclusion].

Non Verticillium longisporum (Stark) Karapappa et al., Mycol. Res. 101: 1293 (1997).

Non Akanthomyces longisporus B. Huang et al., Mycosystema 19: 172 (2000).

Akanthomyces lecanii (Zimm.) Spatafora, Kepler \& B. Shrestha, comb. nov.

MycoBank MB820881

Basionym: Cephalosporium lecanii Zimm., Teysmania 9: 241 (1899).

Synonyms: Verticillium lecanii (Zimm.) Viégas, Revista Inst. Café Estado São Paulo 14: 754 (1939).

Lecanicillium lecanii (Zimm.) Zare \& W. Gams, Nova Hedwigia 73: 10 (2001).

Torrubiella confragosa Mains, Mycologia 41: 305 (1949).

Cordyceps confragosa (Mains) G.H. Sung et al., Stud. Mycol. 57: 49 (2007).

Hirsutella confragosa Mains, Mycologia 41: 303 (1949).

For further synonyms see Zare \& Gams (2001).

Akanthomyces muscarius (Petch) Spatafora, Kepler \& B. Shrestha, comb. nov.

MycoBank MB820861

Basionym: Cephalosporium muscarium Petch, Naturalist (Hull) 56: 102 (1931).

Synonyms: Lecanicillium muscarium (Petch) Zare \& W. Gams, Nova Hedwigia 73: 13 (2001).

Cephalosporium aphidicola Petch, Trans. Brit. Mycol. Soc. 16: 71 (1931).

Verticillium hemileiae Bouriquet, Encycl. Mycol. 12: 155 (1946).

For further synonyms see Zare \& Gams (2001).

Akanthomyces sabanensis (J.S. Chiriví-Salomón et al.) J.S. Chiriví-Salomón, T. Sanjuan \& S. Restrepo, comb. nov.

MycoBank MB820862

Basionym: Lecanicillium sabanense J.S. Chiriví-Salomón et al., Phytotaxa 234: 68 (2015).

Akanthomyces tuberculatus (Lebert) Spatafora, Kepler \& B. Shrestha, comb. nov.

MycoBank MB820863

Basionym: Akrophyton tuberculatum Lebert, Z. Wiss. Zool. 9: 448 (1858).

Synonyms: Cordyceps tuberculata (Lebert) Maire, Bull. Soc. Hist. Nat. Afrique N. 8: 165 (1917).

Isaria pistillariiformis Pat., Bull. Soc. Mycol. Fr. 9: 163 (1893); as "pistillariaeformis".

Insecticola pistillariiformis (Pat.) Mains, Mycologia 42: 579 (1950); as "pistillariaeformis".

Akanthomyces pistillariiformis (Pat.) Samson \& H.C. Evans, Acta Bot. Neerl. 23: 29 (1974).

Ascopolyporus Möller, Bot. Mitt. Tropen 9: 300 (1901).

Type: Ascopolyporus polychrous Möller, Bot. Mitt. Tropen 9: 300 (1901).

Ascopolyporus is a genus containing seven species, represented in this study by the type $A$. polychrous and A. villosus. These two species are strongly supported as monophyletic, however their relationship to other taxa in Cordycipitaceae remains poorly resolved. Sexual or asexual morphologies have been observed in individual collections of Ascopolyporus, and they co-occur in some species (Bischoff et al. 2005). In the sexual form perithecia are produced in a dense hyphal mat directly on top of the scale insect host, and the appearance is similar to that of species in Hypocrella or Moelleriella in Clavicipitaceae. Ascopolyporus species produce multiseptate conidia, a feature also found in Hyperdermium. Ascopolyporus shares another characteristic with some species in Hypocrella in the apparent utilization of plant resources via the scale insect cadaver to attain sizes greatly in excess of the original host (Hywel-Jones \& Samuels 1998, Bischoff et al. 2005, Chaverri et al. 2008).

Beauveria Vuill., Bull. Soc. Bot. France 59: 40 (1912).

Type: Beauveria bassiana (Bals.-Criv.) Vuill., Bull. Soc. Bot. France 59: 40 (1912).

The recognition of Beauveria as a genus separate from Cordyceps is a significant change for Cordycipitaceae; their respective type species are not congeneric. The morphological features that unite species of Beauveria have proved remarkably durable over time and no isolates described from other asexually typified genera are known in this clade. Direct links between species of Beauveria and cordyceps-like sexual morphs are well established from molecular data and culture-based experiments, including $B$. bassiana (Li et al. 2001, Huang et al. 2002), B. brongniartii (Shimizu et al. 1988), and B. sungii (Shrestha et al. 2014a). The host range for the asexual morphs is extensive, infecting many insect species across multiple orders (de Faria \& Wraight 2007) as well as being isolated from soil and as foliar endophytes (Vega et al. 2009). The sexual morphs are known from Coleoptera, Lepidoptera, Orthoptera, and Phasmatodea, and here we also describe a new species from Blattodea, expanding the known host range of sexual morphs of Beauveria.

Beauveria acridophila (T. Sanjuan \& Franco-Mol.) T. Sanjuan, B. Shrestha, Kepler \& Spatafora, comb. nov.

MycoBank MB820883

Basionym: Cordyceps acridophila T. Sanjuan \& Franco-Mol., Mycologia 106: 268 (2014). 

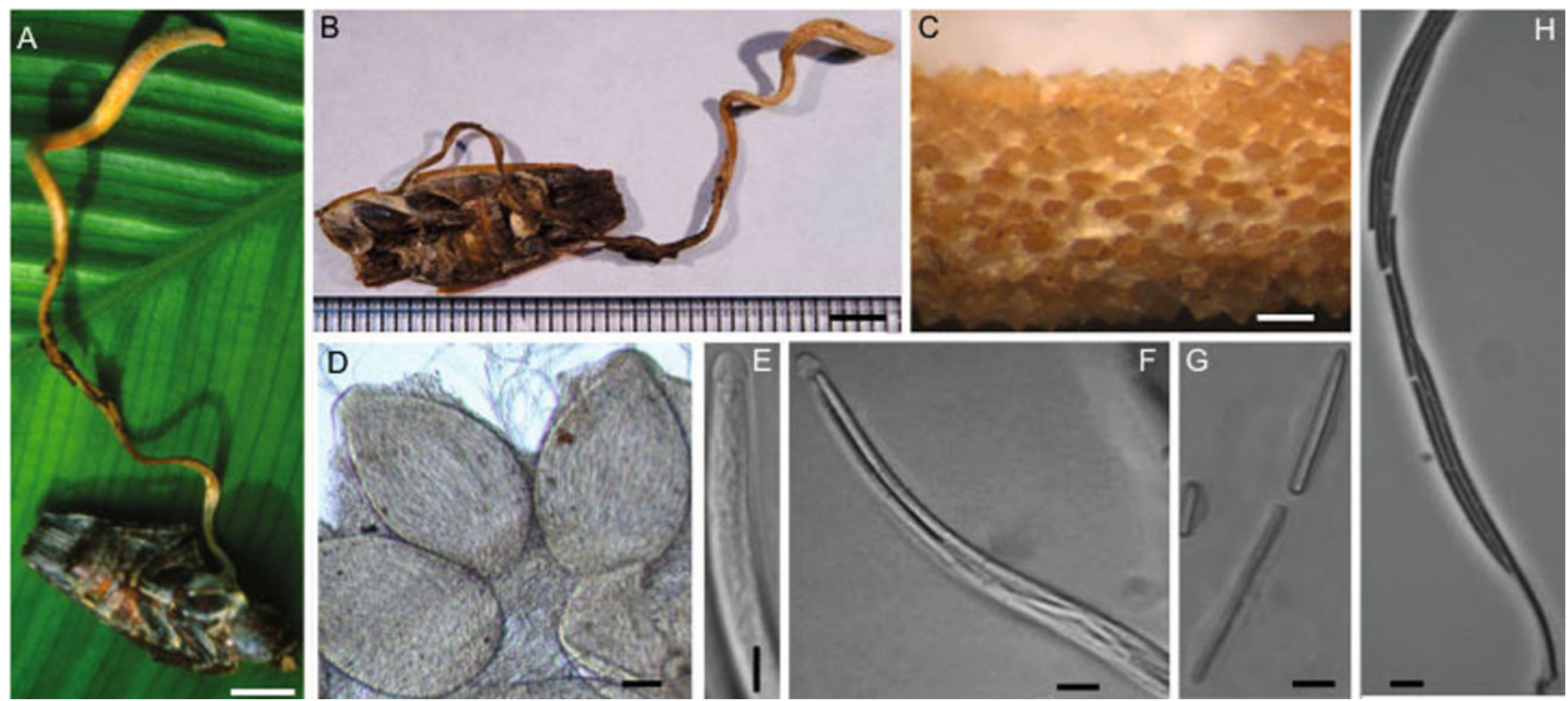

Fig. 2. Beauveria blattidicola (MCA1727 - holotype). A. Fresh stroma on cockroach. B. Dried stroma on cockroach. C. Stroma with partially immersed perithecia. D. Perithecia. E. Ascus showing prominent ascus cap. F. Ascus showing cylindrical ascospores. G. Part-spores. H. End of ascus showing ascus foot. Bars: $A-B=4 \mathrm{~mm} ; C=150 \mu \mathrm{m} ; \mathrm{D}=50 \mu \mathrm{m} ; \mathrm{E}-\mathrm{H}=5 \mu \mathrm{m}$.

Beauveria bassiana (Bals.-Criv.) Vuill., Bull. Soc. Bot. Fr. 59: 40 (1912).

Basionym: Botrytis bassiana Bals.-Criv., Linnaea 10: 611 (1835).

Synonyms: Spicaria bassiana (Bals.-Criv.) Vuill., Bull. Soc. Sc. Nancy, ser. 3, 10: 153 (1910).

Penicillium bassianum (Bals.-Criv.) Biourge, Cellule 33: 101 (1923).

Cordyceps bassiana Z.Z. Li et al., Chin. Sci. Bull. 9: 751 (2001).

Beauveria blattidicola M. Chen, Aime, T.W. Henkel \& Spatafora, sp. nov. (Fig. 2)

MycoBank MB821050

Etymology: The species epithet refers to the fungus' occurrence on the host insect family Blattidae.

Diagnosis: Similar in host association to Ophiocordyceps blattarioides but differs in the yellow-orange fleshy stromata, long, sinuous stipe, and cylindrical to narrowly clavate fertile region with partially immersed perithecia.

Type: Guyana: Region 8, Potaro-Siparuni: Pakaraima Mountains, Upper Potaro River Basin, within a $4 \mathrm{~km}$ radius of Potaro base camp at $5^{\circ} 18^{\prime} 04.8^{\prime \prime} \mathrm{N}, 59^{\circ} 54^{\prime} 40.4^{\prime \prime} \mathrm{W}, 710-750$ m elev.; on adult cockroach, 31 May, 2001, M.C. Aime MCA 1727 (BRG - holotype).

Description: Stromata solitary or paired, unbranched, arising from head or thorax of infected adult of cockroach, with fleshy texture, total length $50-60(-90) \mathrm{mm}$ long; stalk $0.8-1.5 \mathrm{~mm}$ broad, light yellow (4A3-4A4); fertile area apical, cylindrical to narrowly clavate, $6-8 \times 1.2-1.4 \mathrm{~mm}$, yellowish orange (4A74A8). Perithecia partially immersed, darker concolorous, interspersed with white mycelial wefts, presented at right angle to the surface of stroma, in longitudinal section oval to ovoid, $250-370 \times 110-250 \mu \mathrm{m}$. Asci hyaline, cylindrical, $170-300 \times 3-4 \mu \mathrm{m}$, with a prominent apical cap 3.0-4.0 $\mu \mathrm{m}$ diam. Ascospores filiform, nearly as long as the asci, smooth, hyaline, distinct irregularly multiseptate, not easily breaking into part-spores. Part-spores $6.0-23.0 \times 1.0 \mu \mathrm{m}$, cylindrical with truncate ends.

\section{Known distribution: Guyana.}

Additional specimens examined: Guyana: Region 8, PotaroSiparuni: Pakaraima Mountains, Upper Potaro River Basin, within a $4 \mathrm{~km}$ radius of Potaro base camp at $5^{\circ} 18^{\prime} 04.8^{\prime \prime} \mathrm{N}, 59^{\circ} 54^{\prime} 40.4^{\prime \prime} \mathrm{W}$, 710-750 m elev., on adult cockroach adhered to leaf in litter, 12 June 2000, M.C. Aime MCA 1203 (PUL); on adult cockroach in leaf litter, 14 July 2000, T.W. Henkel TH 7645 (HSC); on adult cockroach, 25 May 2001, M.C. Aime MCA 1628 (PUL); on adult cockroach, 7 June 2001, M.C. Aime MCA 1814 (PUL); on adult cockroach in leaf litter, 24 July 2003, T.W. Henkel TH 8607 (HSC); on adult cockroach on mineral soil below leaf litter, 17 July 2009, T.W. Henkel TH 9049 (OSC); on adult cockroach, partially buried in litter, 27 May 2010, M.C. Aime \& L. Williams MCA 4043 (PUL); on adult cockroach, 9 June 2012, M.C. Aime MCA 4883 (PUL).

GenBank: MCA1727 MF416593, MF416539, MF416483, MF416640; MCA1814 MF416594, MF416540, MF416484, MF416641

Commentary: Species of Cordyceps s. lat. infecting cockroaches have rarely been collected in nature. Only two cockroach-associated species are recorded in the literature: Ophiocordyceps blattarioides (Sanjuan et al. 2015) and O. blattae (Petch 1924, 1931), both classified in Ophiocordycipitaceae. The Neotropical O. blattarioides is associated with adult Blattodea, and is closely related to the morphologically similar adult Orthoptera-associated O. amazonica (Sanjuan et al. 2015). 
Ophiocordyceps blattae was described originally from Sri Lanka (as Cordyceps blattae), but the species remains poorly known, as sequence data are lacking, and current taxonomic concepts are based entirely on the original description and drawings of Petch (1924). Based on the possession of ophio-ascospores and clavate asci with reduced apices, $O$. blattae is likely closely related to O. unilateralis (Petch 1931) and is the type of the genus Ophiocordyceps (Sung et al. 2007). Beauveria blattidicola is easily distinguished from both $O$. blattarioides and $O$. blattae by the combination of yellow to yellowish orange fleshy stromata, the long and sinuous stalk, cylindrical to narrowly clavate fertile region, and partially immersed perithecia typical of other sexual morphs of Beauveria. Molecular data also strongly support the placement of $B$. blattidicola in Beauveria (Fig. 1). While $B$. blattidicola cultures are currently unavailable, its phylogenetic placement predicts a Beauveria-type asexual morph.

Beauveria brongniartii (Sacc.) Petch, Trans. Brit. Mycol. Soc. 10: 249 (1926).

Basionym: Botrytis brongniartii Sacc., Syll. Fung. 10: 540 (1892).

Synonym: Cordyceps brongniartii Shimazu, Trans. Mycol. Soc. Japan 29: 328 (1989).

Beauveria diapheromeriphila (T. Sanjuan \& S. Restrepo) T. Sanjuan, B. Shrestha, Kepler \& Spatafora, comb. nov.

MycoBank MB820882

Basionym: Cordyceps diapheromeriphila T. Sanjuan \& S. Restrepo, Mycologia 106: 270 (2014).

Beauveria locustiphila (Henn.) B. Shrestha, Kepler \& Spatafora, comb. nov.

MycoBank MB820884

Basionym: Cordyceps locustiphila Henn., Hedwigia 43: 246 (1904).

Beauveria scarabaeidicola (Kobayasi) S.A. Rehner \& Kepler, comb. nov.

MycoBank MB820891

Basionym: Cordyceps scarabaeidicola Kobayasi, Bull. Natl. Sci. Mus. Tokyo, B 2: 137 (1976); as "scarabaeicola".

Synonym: Beauveria sungii S.A. Rehner \& R.A. Humber, Mycologia 103: 1070 (2011).

Beauveria staphylinidicola (Kobayasi \& Shimizu) B. Shrestha, Kepler \& Spatafora, comb. nov.

MycoBank MB820895

Basionym: Cordyceps staphylinidicola Kobayasi \& Shimizu, Bull. Natl. Sci. Mus. Tokyo, B 8: 88 (1982); as "staphylinidaecola".

Blackwellomyces Spatafora \& Luangsa-ard, gen. nov.

\section{MycoBank MB820864}

Etymology: This genus is named for Meredith Blackwell and honours her invaluable contributions to our knowledge of insect-associated fungi.
Diagnosis: Blackwellomyces is the least inclusive genuslevel clade that includes the species $B$. cardinalis and $B$. pseudomilitaris. Blackwellomyces is diagnosed by the unique characters of the ascospore, which have irregularly spaced septa and do not disarticulate into part-spores at maturity.

Type: Blackwellomyces cardinalis (G.H. Sung \& Spatafora) Spatafora \& Luangsa-ard 2017.

Description: Sexual morph: Stromata solitary or multiple, simple or branched. Stipe fleshy, orange to red, cylindrical to enlarging apically, $4-50 \times 0.5-3.0 \mathrm{~mm}$. Fertile area terminal, cylindrical, fusiform to clavate to irregularly shaped, 2-9 × 1-4 mm. Perithecia crowded, loosely embedded, ordinal in orientation, elliptical to fusiform to obclavate. Asci 8-spored, hyaline, cylindrical, possessing a prominent apex. Ascospores smooth, filiform, hyaline, irregularly multiseptate, not fragmenting into part-spores.

Asexual morph: Cultures moderately fast growing in PDA and may turn the media red. Aerial mycelium is whitish to whitish yellow and the reverse side of cultures is red or cream. Conidiogenous cells phialides, solitary or in whorls of 2 or 3 , swollen at the base or slightly flask-shaped, wider near the base and tapering at the apex. Conidia hyaline, aseptate, ellipsoidal to elliptical, in some species produced in sympodially imbricate chains. Asexual morphs have been described as similar to species in Clonostachys, Hirsutella, Isaria, and Mariannaea.

Hosts: On larva of Lepidoptera.

Distribution: Southeastern USA, eastern China, Japan, Korea, and Thailand.

Commentary: The species placed here are supported as a distinct clade and separate from other genera of Cordycipitaceae based on the placement of their type species. We describe these taxa as Blackwellomyces on the basis of their phylogenetic novelty and irregularly septate ascospores that do not disarticulate into part-spores. This contrasts with other members of the family in which septation and disarticulation is common.

Blackwellomyces cardinalis (G.H. Sung \& Spatafora) Spatafora \& Luangsa-ard, comb. nov. MycoBank MB820865

Basionym: Cordyceps cardinalis G.H. Sung \& Spatafora, Mycologia 96: 660 (2004).

Blackwellomyces pseudomilitaris (Hywel-Jones \& Sivichai) Spatafora \& Luangsa-ard, comb. nov.

MycoBank MB820866

Basionym: Cordyceps pseudomilitaris Hywel-Jones \& Sivichai, Mycol. Res. 98: 940 (1994).

Cordyceps Fr., Observ. Mycol. 2: 316 [cancellans] (1818), nom. cons.

Type: Cordyceps militaris (L.) Fr., Observ. Mycol. 2: 317 [cancellans] (1818). 
In this analysis, many species of Cordyceps, including the type, are resolved as a well-supported clade interspersed with genera described originally for asexual morphs, including Evlachovaea, Isaria, and Microhilum (Fig. 1). Additionally, our analysis indicates this core Cordyceps is not monophyletic with C. cardinalis and C. pseudomilitaris, a result consistent with Sung et al. (2007); the latter two species are proposed in the new genus Blackwellomyces here (see above). Within the core Cordyceps clade, internal relationships are generally well-supported, giving rise to a phylogenetic structure that roughly corresponds to stromatal colour (red to orange vs. white to yellow). A thorough review of the taxonomic history of Cordyceps was provided by Shrestha et al. (2014b), who concluded that Cordyceps is the oldest accepted generic name in this clade and is typified by a sexual morph. Based on the cylindrical shape of the stroma, pre-Linnaean literature of the $17^{\text {th }}$ and early $18^{\text {th }}$ century had recorded $C$. militaris, the type species of Cordyceps, under the old but obsolete generic names Fungus and Fungoides (Shrestha et al. 2014b). The species was transferred to Clavaria by Linnaeus (1753). Clavaria militaris was then transferred to the ascomycete genus Sphaeria (now rejected in favour of Hypoxylon), a classification that was followed until the early $19^{\text {th }}$ century (Shrestha et al. 2014b). The genus Cordyceps was established (Fries 1818, Link 1833) and over the years was circumscribed to include pathogens of more than 12 insect orders and the fungal genera Elaphomyces and Claviceps (Kobayasi 1941, Mains 1958, Sung et al. 2007, Kepler et al. 2012, Araújo \& Hughes 2016, Shrestha et al. 2016). This generic concept of Cordyceps stood for approximately 200 years until the polyphyletic nature of Cordyceps as it had been understood by Kobayasi and Mains was revealed (Sung et al. (2007).

Three monotypic generic names are now considered to be synonyms of Cordyceps. Our data confirmed the findings of Humber et al. (2013) who demonstrated that the type species of the monotypic Evlachovaea, E. kintrischica, is a synonym of Isaria (see below). Similarly, the type species of Microhilum, M. oncoperae, known to have a Cordyceps sexual morph, was nested within Cordyceps, as is the type species of Phytocordyceps, $P$. ninchukispora.

The generic name Isaria is the oldest available name for the entire group of taxa considered here, including Cordyceps. However, the concept of Isaria has a long and convoluted history, with many changes of status and differences of opinion in how the name should be applied (e.g. Hodge et al. 2005, Gams et al. 2005). Petch (1934) concluded the name was too confusing to use and suggested it be applied to a subgenus of Spicaria. However, Hodge et al. (2005) lectotypified Isaria sensu Fries using an illustration of $I$. farinosa that appeared in the original description of this species as Ramaria farinosa (Holmskjold 1781). Gams et al. (2005) proposed the use of Isaria for Paecilomyces sect. Isarioidea, now also regarded as a synonym of Cordyceps. Entomogenous species morphologically similar to Isaria can be found distributed throughout Hypocreales (Luangsa-ard et al. 2004), and here are shown to be polyphyletic within Cordycipitaceae. The ex-epitype isolate of $I$. farinosa (CBS 111113, Gams et al. 2005) is here determined to belong within Cordyceps. We therefore propose the rejection of Isaria in favour of Cordyceps owing to the confusion surrounding the application of Isaria. Additionally, rejecting Cordyceps would be disruptive to a large user community while the name Isaria is not as widely used. Species of Isaria are herein integrated into the monophyletic application of Cordyceps. The diversity of species infecting cicada nymphs complicates the transfer of $I$. cicadae, and will be addressed in a subsequent paper focused on the group.

Cordyceps amoene-rosea (Henn.) Kepler, B. Shrestha \& Spatafora, comb. nov.

MycoBank MB820975

Basionym: Isaria amoene-rosea Henn., Hedwigia 41: 66 (1902).

Synonym: Paecilomyces amoeneroseus (Henn.) Samson, Stud. Mycol. 6: 37 (1974).

Cordyceps cateniannulata (Z.Q. Liang) Kepler, B. Shrestha \& Spatafora, comb. nov.

MycoBank MB820976

Basionym: Paecilomyces cateniannulatus Z.Q. Liang, Acta Phytopathol. Sin. 11: 10 (1981).

Synonym: Isaria cateniannulata (Z.Q. Liang) Samson \& Hywel-Jones, Mycol. Res. 109: 588 (2005).

Cordyceps cateniobliqua (Z.Q. Liang) Kepler, B. Shrestha \& Spatafora, comb. nov.

MycoBank MB820977

Basionym: Paecilomyces cateniobliquus Z.Q. Liang, Acta Phytopathol. Sin. 11: 9 (1981).

Synonym: Isaria cateniobliqua (Z.Q. Liang) Samson \& HywelJones, Mycol. Res. 109: 588 (2005).

Cordyceps coleopterorum (Samson \& H.C. Evans) Kepler, B. Shrestha \& Spatafora, comb. nov.

MycoBank MB820978

Basionym: Paecilomyces coleopterorum Samson \& H.C. Evans, Stud. Mycol. 6: 47 (1974).

Synonym: Isaria coleopterorum (Samson \& H.C. Evans) Samson \& Hywel-Jones, Mycol. Res. 109: 588 (2005); as "coleopterora".

Cordyceps farinosa (Holmsk.) Kepler, B. Shrestha \& Spatafora, comb. nov.

MycoBank MB820979

Basionym: Ramaria farinosa Holmsk., K. Danske Vidensk. Selsks. Skr., Nye Samling 1: 279 (1781).

Synonyms: Clavaria farinosa (Holmsk.) Dicks., Fasc. Pl. Crypt. Brit. 2: 25 (1790).

Isaria farinosa (Holmsk.) Fr., Syst. Mycol. 3: 271 (1832); nom. sanct.

Corynoides farinosa (Holmsk.) Gray, Nat. Arr. Brit. PI. 1: 654 (1821).

Spicaria farinosa (Holmsk.) Vuill., Bull. Soc. Mycol. France 27: 76 (1911).

Penicillium farinosum (Holmsk.) Biourge, Cellule 33: 102 (1923).

Paecilomyces farinosus (Holmsk.) A.H.S. Br. \& G. Sm., Trans. Brit. Mycol. Soc. 40: 50 (1957). 
Cordyceps fumosorosea (Wize) Kepler, B. Shrestha \& Spatafora, comb. nov.

MycoBank MB820980

Basionym: Isaria fumosorosea Wize, Bull. Int. Acad. Sci. Cracovie, Cl. Sci. Math. Nat.: 721 (1905) ["1904"].

Synonyms: Spicaria fumosorosea (Wize) Vassiljevski, Morbi Plant. 18:146 (1929).

Paecilomyces fumosoroseus (Wize) A.H.S. Br. \& G. Sm., Trans. Brit. Mycol. Soc. 40: 67 (1957).

Cordyceps ghanensis (Samson \& H.C. Evans) Kepler, B. Shrestha \& Spatafora, comb. nov.

MycoBank MB820981

Basionym: Paecilomyces ghanensis Samson \& H.C. Evans, Stud. Mycol. 6: 46 (1974).

Synonym: Isaria ghanensis (Samson \& H.C. Evans) Samson \& Hywel-Jones, Mycol. Res. 109: 588 (2005).

Cordyceps javanica (Frieder. \& Bally) Kepler, B. Shrestha \& Spatafora, comb. nov.

MycoBank MB820982

Basionym: Spicaria javanica Frieder. \& Bally, Meded. Koffiebessenboeboek-Fonds 6: 146 (1923).

Synonyms: Paecilomyces javanicus (Frieder. \& Bally) A.H.S. Br. \& G. Sm., Trans. Brit. Mycol. Soc. 40: 65 (1957).

Isaria javanica (Frieder. \& Bally) Samson \& Hywel-Jones, Mycol. Res. 109: 588 (2005).

Cordyceps kintrischica (B.A. Borisov \& Tarasov) Kepler, B. Shrestha \& Spatafora, comb. nov.

MycoBank MB820983

Basionym: Evlachovaea kintrischica B.A. Borisov \& Tarasov, Mikol. Fitopatol. 33: 250 (1999).

Cordyceps locusticola (Z.Q. Liang et al.) Kepler, B. Shrestha \& Spatafora, comb. nov.

MycoBank MB820984

Basionym: Isaria locusticola Z.Q. Liang et al., Mycotaxon 105: 31 (2008).

Cordyceps oncoperae (H.Y. Yip \& A.C. Rath) P.J. Wright, J. Invert. Path. 64: 146 (1994).

MycoBank MB363549

Basionym: Microhilum oncoperae H.Y. Yip \& A.C. Rath, J. Invert. Path. 53: 362 (1989).

Cordyceps poprawskii (Cabanillas et al.) Kepler, B. Shrestha \& Spatafora, comb. nov.

MycoBank MB820985

Basionym: Isaria poprawskii Cabanillas et al., Mycoscience 54: 162 (2013).

Cordyceps tenuipes (Peck) Kepler, B. Shrestha \& Spatafora, comb. nov.

MycoBank MB820986

Basionym: Isaria tenuipes Peck, Ann. Rep. N.Y. St. Mus. Nat. Hist. 31: 44 (1879).

Synonyms: Paecilomyces tenuipes (Peck) Samson, Stud. Mycol. 6: 49 (1974).
Cordyceps takaomontana Yakush. \& Kumaz., Sci. Rep. Tokyo Bunrika Daig., B 5: 108 (1941).

\section{Engyodontium de Hoog, Persoonia 10: 53 (1978).}

Type: Engyodontium parvisporum (Petch) de Hoog, Persoonia 10: 53 (1978).

Engyodontium was erected by de Hoog (1978) to accommodate the type species, E. parvisporum, and E. album, the latter species was formerly classified in Beauveria, and most recently placed in Parengyodontium (see below). Gams et al. (1984) added four more species. The genus is restricted here to species with cobweb-like colonies that produce dense clusters of denticles on elongated rachides. Conidia are hyaline and globose to subglobose. No sexual reproductive morph has been linked to Engyodontium. Molecular phylogenetic analyses based on $E$. aranearum support the distinction from Beauveria, but additional study of the type species is required. Species are isolated from soil and arthropod cadavers, and as opportunistic cutaneous and subcutaneous infections of animals including humans.

Gibellula Cavara, Atti Ist. Bot. R. Univ. Pavia, 2 ser. 3: 347 (1894).

Type: Gibellula pulchra Cavara, Atti Ist. Bot. Univ. Lab. Crittog. Pavia 3: 347 (1894).

The genus Gibellula is recognized here for spiderpathogenic fungi that produce primarily synnematous, aspergillus-like conidiophores with terminal vesicles, which give rise to phialides produced on metulae. Molecular phylogenies place all sampled Gibellula species in a single clade along with torrubiella-like sexual morphs. Torrubiella has been shown to be polyphyletic, including astipitate taxa throughout Cordycipitaceae and Hypocreales. The status of Torrubiella is complicated further by the uncertain phylogenetic placement of the type species, $T$. aranicida. The original description indicated an asexual morphology that more closely approximates Lecanicillium or Simplicillium. Further, the production of scattered perithecia directly from the host, rather than aggregated on a subiculate pad, do not suggest inclusion of Gibellula in Torrubiella. Rather, the genus Torrubiella is regarded as a synonym of Akanthomyces here based on the perithecial arrangement and lack of a subiculum in the type specimen (discussed under Akanthomyces). The genus Granulomanus, based on $G$. aranearum which is linked to the sexually typified T. albolanata, was regarded as a synonym of Gibellula by Humber \& Rombach (1987), but no molecular data exist to confirm or refute this suggestion.

Gibellula arachnophila (Ditmar) Vuill., Bull. Séanc. Soc. Sci. Nancy, sér. 3 11: 156 (1910).

Basionym: Isaria arachnophila Ditmar, in Sturm, Deutschl. Fl., 3 Abt. (Pilze Deutschl.) 1(4): tab. 55 (1817).

Synonyms: Hymenostilbe arachnophila (Ditmar) Petch, Naturalist (Hull) 56: 249 (1931). 
Synsterigmatocystis arachnophila Costantin ex Vuill., Bull. Soc. Mycol. France 27: 81 (1911).

Gibellula arachnophila f. macropus Vuill., Bull. Soc. Mycol. France 36: 41 (1920).

Torrubiella arachnophila f. alba Kobayasi \& Shimizu, Kew Bull. 31: 561 (1977).

Cordyceps arachnophila J.R. Johnst., Bull. Puerto Rico Insula Exp. Sta. 10: 23 (1915).

Torrubiella arachnophila (J.R. Johnst.) Mains, Mycologia 42: 316 (1950).

Gibellula aranearum P. Syd., Just's Bot. Jahresber. 57: 321 (1922).

Synonym: Torrubiella gibellulae Petch, Ann. Mycol 30: 391 (1932).

Gibellula clavata Samson \& H.C. Evans, Mycologia 84: 306 (1992).

Synonym: Torrubiella clavata Samson \& H.C. Evans, Mycologia 84: 306 (1992).

Gibellula dabieshanensis B. Huang et al., Mycosystema 17: 110 (1998).

Synonym: Torrubiella dabieshanensis B. Huang et al., Mycosystema 17: 110 (1998).

Gibellula dimorpha Tzean et al., Mycol. Res. 102: 1350 (1998).

Synonym: Torrubiella dimorpha Tzean et al., Mycol. Res. 102: 1350 (1998).

Gibellula globosa Kobayasi \& Shimizu, Bull. Natn. Sci. Mus. Tokyo, B 8: 45 (1982).

Synonym: Torrubiella globosa Kobayasi \& Shimizu, Bull. Natn. Sci. Mus. Tokyo, B 8: 45 (1982).

Gibellula globosostipitata Kobayasi \& Shimizu, Bull. Natn. Sci. Mus. Tokyo, B 8: 49 (1982).

Basionym: Torrubiella globosostipitata Kobayasi \& Shimizu, Bull. Natn. Sci. Mus. Tokyo, B 8: 49 (1982).

Gibellula leiopus (Vuill. ex Maubl.) Mains, Mycologia 42: 313 (1950).

Basionym: Gibellula arachnophila f. leiopus Vuill. ex Maubl., Bull. Soc. Mycol. France 36: 42 (1920).

Torrubiella arachnophila var. leiopus Mains, Mycologia 42: 318 (1950).

Torrubiella leiopus (Mains) Kobayasi \& Shimizu, Kew Bull. 31: 564 (1977); as "pleiopus".

Gibellula pulchra Cavara, Atti Ist. bot. R. Univ. Pavia, 2 sér. 3: 347 (1894).

Synonyms: Torrubiella arachnophila var. pulchra Mains, Mycologia 42: 316 (1950).

Torrubiella pulchra (Mains) Koval, Klavitsipital'nye Griby SSSR: 71 (1984).

Hevansia Luangsa-ard, Hywel-Jones \& Spatafora, gen. nov.

MycoBank MB820885
Etymology: This genus is named for Harry C Evans and honours his invaluable contributions to our knowledge of insect associated fungi.

Diagnosis: Hevansia is the least inclusive genus-level clade that includes $H$. novoguineensis and $H$. nelumboides. Hevansia is diagnosed by the immersed perithecia, an Akanthomyces-like asexual morph, and parasitism on spiders.

Type: Hevansia novoguineensis (Samson \& B.L. Brady) Luangsa-ard et al. 2017 (see below)

Description: Sexual morph: Stroma arising from dorsal abdomen, stipe 1-2 mm, fertile part ca $1 \mathrm{~mm}$, white to cream, or in astipitate perithecial cushions surrounding host abdomen or sides of legs. Perithecia immersed, crowded at stipe apex or in cushions, few $(<5)$ to numerous $(30+)$, narrowly ovoid or sickle-shaped. Asci 8-spored, ascospores filiform, hyaline, whole, or disarticulating into part-spores.

Asexual morph: Stroma white, cream-yellow, brown or grey mycelium completely covering host. Synnemata erect, simple or branched, solitary to numerous, cylindrical to clavate, cream to ash-grey or brownish white. In some species 2-4 prominent synnemata up to $6 \mathrm{~mm}$ long interspersed with numerous tiny synnemata scattered over host. Phialides in a monolayer, sparsely scattered or crowded, on a basal cell or arising from lateral cells, usually single, occasionally two or three on lateral basal cell, smooth-walled, cylindrical, globose, obovoid, obpyriform or ellipsoid, terminating in short but distinct neck or tapering into a long neck. Conidia catenate, usually one-celled, smooth-walled, hyaline, clavate, cylindrical, cymbiform, fusiform to narrowly obclavate. Colony on PDA white front, reverse cream, orange to pale red, some species with pale wine-red pigment on the agar.

Hosts: On spiders on the underside of leaves of forest plants.

Distribution: Primarily in tropical regions globally, although specimens are known from temperate regions.

Commentary: A biphyletic split is observed among the astipitate species previously considered in Torrubiella with asexual morphs in Gibellula forming a clade of species pathogenic on spiders. Another clade includes species on spiders in the tropics, many of which were previously considered members of Akanthomyces. These species constitute the new genus Hevansia described here. Placement of the spider pathogen C. nelumboides in this genus demonstrates the diverse morphology of Hevansia species, as C. nelumboides produces perithecia in a disc sitting atop a well-formed stipe.

Hevansia arachnophila (Petch) Luangsa-ard, HywelJones \& Spatafora, comb. nov.

MycoBank MB820886

Basionym: Trichosterigma arachnophilum Petch, Trans. Brit. Mycol. Soc. 8: 215 (1923); as "arachnophila".

Synonyms: Hirsutella arachnophila (Petch) Petch, Trans. Brit. Mycol. Soc. 9: 93 (1923). 
Akanthomyces arachnophilus (Petch) Samson \& H.C. Evans, Acta Bot. Neerl. 23: 33 (1974).

Torrubiella flava Petch, Trans. Brit. Mycol. Soc. 9: 127 (1923).

Hevansia cinerea (Hywel-Jones) Luangsa-ard, HywelJones \& Spatafora, comb. nov.

MycoBank MB820887

Basionym: Akanthomyces cinereus Hywel-Jones, Mycol. Res. 100: 1068 (1996).

Hevansia koratensis (Hywel-Jones) Luangsa-ard, Hywel-Jones \& Spatafora, comb. nov.

MycoBank MB820888

Basionym: Akanthomyces koratensis Hywel-Jones, Mycol. Res. 100: 1067 (1996).

Hevansia longispora (B. Huang et al.) Luangsa-ard, Hywel-Jones \& Spatafora, comb. nov.

MycoBank MB820889

Basionym: Akanthomyces longisporus B. Huang et al., Mycosystema 19: 172 (2000).

Hevansia nelumboides (Kobayasi \& Shimizu) Luangsaard, Hywel-Jones \& Spatafora, comb. nov.

MycoBank MB820890

Basionym: Cordyceps nelumboides Kobayasi \& Shimizu, Kew Bull. 31: 557 (1977).

Hevansia novoguineensis (Samson \& B.L. Brady) Luangsa-ard, Hywel-Jones \& Spatafora, comb. nov. MycoBank MB820892

Basionym: Akanthomyces novoguineensis Samson \& B.L. Brady, Trans. Brit. Mycol. Soc. 79: 571 (1982).

Hevansia ovolongata (L.S. Hsieh et al.) Luangsa-ard, Hywel-Jones \& Spatafora, comb. nov.

MycoBank MB820893

Basionym: Akanthomyces ovalongatus L.S. Hsieh et al., Mycologia 89: 321 (1997).

Hevansia websteri (Hywel-Jones) Luangsa-ard, Hywel-Jones \& Spatafora, comb. nov.

MycoBank MB820894

Basionym: Akanthomyces websteri Hywel-Jones, Mycol. Res. 100: 1068 (1996).

Hyperdermium J.F. White et al., Mycologia 92: 910 (2000).

Type: Hyperdermium caulium (Berk. \& M.A. Curtis) Chaverri \& K.T. Hodge, Stud. Mycol. 60: 63 (2008) [syn. H. bertonii (Speg.) J.F. White et al., Mycologia 92: 910 (2000)].

Hyperdermium was erected by Sullivan et al. (2000) for Epichloë bertonii and H. pulvinatum, which parasitize scale insects in the tropics. Stromata are flattened or pulvinate and vary in colour from white to orange. Perithecia are immersed to sub-immersed with asci and ascospores characteristic of Cordycipitaceae, but are unique in producing multiseptate conidia. Hyperdermium bertonii, the type species of the genus, was found to have an earlier epithet, so the name was corrected to $H$. caulium by Chaverri (et al. 2008). In these analyses $H$. caulium, with $C$. piperis, is placed in an unresolved position.

Parengyodontium C.C. Tsang et al., Med. Mycol. 54: 708 (2016).

Type: Parengyodontium album (Limber) C.C. Tsang et al., Med. Mycol. 54: 709 (2016).

Based on molecular phylogenetic analyses, Parengyodontium was erected by Tsang et al. (2016) to recognize Engyodontium album as a distinct taxon relative to E. parvisporum. To date it is a monotypic genus.

Simplicillium W. Gams \& Zare, Nova Hedwigia 73: 38 (2001).

Type: Simplicillium lanosiniveum (J.F.H. Beyma) Zare \& W. Gams, Nova Hedwigia 73: 39 (2001).

Simplicillium includes species isolated from other fungi and soil environments (Zare \& Gams 2001, Nonaka et al. 2013). Current phylogenetic analyses resolve Simplicillium as the earliest diverging lineage in Cordycipitaceae (Fig. 1). Simplicillium species are morphologically reduced, producing conidia on the tips of long, slender, solitary phialides. No sexual forms have been associated with Simplicillium.

\section{CONCLUSIONS}

In this paper we used a multigene phylogeny (Fig. 1) to guide a taxonomic revision of Cordycipitaceae in compliance with changes to Art. 9 of the ICN to no longer permit the separate naming of fungal morphs (McNeill et al. 2012). The resulting analysis provides the basis for recognition of 11 genera in Cordycipitaceae regardless of life-stage or the associated morphological differences (Fig. 3). The generic name Cordyceps is retained, and we sought to circumscribe that genus in the most inclusive way possible. The ex-epitype isolate of the type of Isaria, I. farinosa, is nested within Cordyceps, yet we recommend the rejection of Isaria, to avoids further splitting of Cordyceps. Here, Beauveria includes the traditional species known from asexual morphs, but also several taxa previously described for sexual morphs in Cordyceps and a new sexually typified species described here. Our approach allows continuity for the use of names of taxa important for biocontrol and historical concepts of diversity for the group. We propose to use the name Gibellula for a clade of spider pathogens, rather than Torrubiella. This decision is based on morphology of the type species, $T$. aranicida, which includes superficial perithecia produced in a scattered manner, and not on a subiculum, and an asexual morph described as more similar to Akanthomyces than Gibellula. Akanthomyces has priority over Torrubiella, although we cannot discount a phylogenetic affinity of Torrubiella to $T$. wallacei or Simplicillium. Therefore, we recommend the rejection of Torrubiella against Akanthomyces. Finally, the use of Lecanicillium is resolved. 

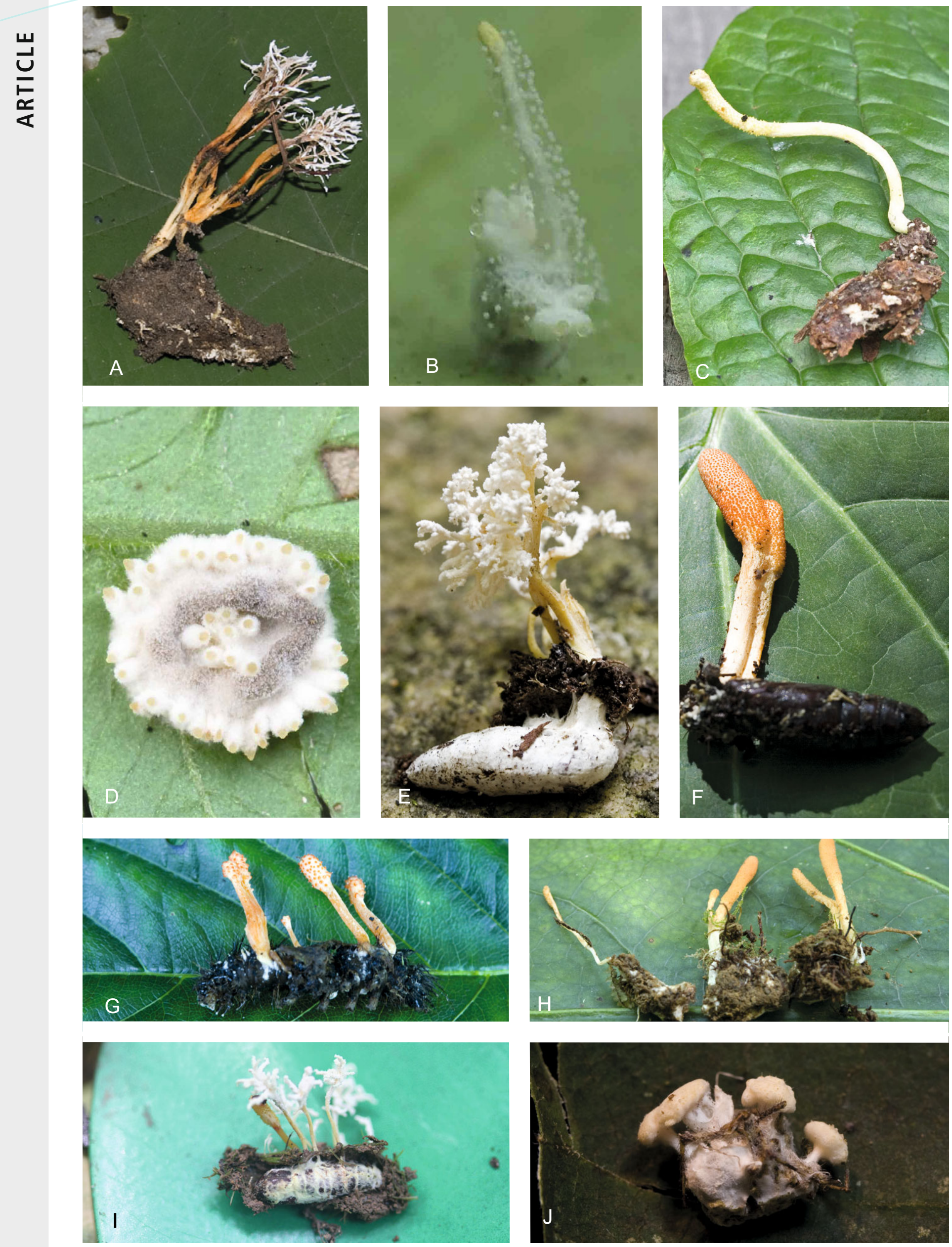
The type, L. lecanii, is nested within Akanthomyces, which has priority over Lecanicillium. We classify several species of Lecanicillium in Akanthomyces. Lecanicillium psalliotae and $L$. fusisporum do not show a strong affinity with other species previously placed in Lecanicillium nor with any other clade in Cordycipitaceae, so these species cannot yet be placed in any named genera.

\section{ACKNOWLEDGEMENTS}

This manuscript is dedicated to our colleague and esteemed mycologist Walter Gams (1934-2017) whose contributions to taxonomy and nomenclature of fungi made this work possible. We would like to acknowledge the following individuals for their contribution to this manuscript: Ryan Woolverton, Yi-jian Yao, Shenghua $\mathrm{Wu}$, and also members of the Japan Society for Vegetable Wasps and Plant Worms. This research was supported by Bioindustry Technology Development Program (316025-05) of IPET (Korea Institute of Planning and Evaluation for Technology in Food, Agriculture, Forestry and Fisheries) of Ministry for Food, Agriculture, Forestry and Fisheries, Korea and the National Center for Genetic Engineering and Biotechnology (BIOTEC), National Science and Technology Development Agency, Thailand. This material is based upon work supported by the National Science Foundation Foundation (DEB-1258162, DEB-0529752 to JWS; DEB-0918591, DEB-1556339 to TWH; OISE-0714106, OISE-0914288 to RMK). Any opinions, findings, and conclusions or recommendations expressed in this material are those of the author(s) and do not necessarily reflect the views of the National Science Foundation. The mention of trade names or commercial products in this publication is solely for the purpose of providing specific information and does not imply recommendation or endorsement by the US Department of Agriculture (USDA); the USDA is an equal opportunity provider and employer.

\section{REFERENCES}

Araújo JPM, Hughes DP (2016) Diversity of entomopathogenic fungi: which groups conquered the insect body? Advances in Genetics 94: 1-39.

Ariyawansa HA, Hyde KD, Jayasiri SC, Buyck B, Chethana KWT, et al. (2015) Fungal diversity notes 111-252-taxonomic and phylogenetic contributions to fungal taxa. Fungal Diversity 75 : 27-274.

Ban S, Azuma Y, Sato H, Suzuki KI, Nakagiri A (2015) Isaria takamizusanensis is the anamorph of Cordyceps ryogamimontana, warranting a new combination, Purpureocillium takamizusanense comb. nov. International Journal of Systematic and Evolutionary Microbiology 65: 2459-2465.

Bischoff JF, Chaverri P, White Jr. JF (2005) Clarification of the host substrate of Ascopolyporus and description of Ascopolyporus philodendrus sp. nov. Mycologia 97: 710-717.
Boudier E (1885) Note sur un nouveau genre et quelques nouvelles especes des Pyrenomycetes. Revue Mycologique 7: 224-227.

Chaverri P, Liu M, Hodge KT (2008) A monograph of the entomopathogenic genera Hypocrella, Moelleriella and Samuelsia gen. nov. (Ascomycota, Hypocreales, Clavicipitaceae), and their aschersonia-like anamorphs in the Neotropics. Studies in Mycology 60: 1-66.

Chiriví-Salomón JS, Danies G, Restrepo S, Sanjuan T (2015) Lecanicillium sabanense sp. nov. (Cordycipitaceae) a new fungal entomopathogen of coccids. Phytotaxa 234: 63-74.

De Faria MR, Wraight SP (2007) Mycoinsecticides and mycoacaricides: a comprehensive list with global coverage and international classification of formulation types. Biological Control 43: 237-256.

De Hoog GS (1978) Notes on some fungicolous hyphomycetes and their relatives. Persoonia 10: 33-81.

Eriksson O (1982) Cordyceps bifusispora spec. nov. Mycotaxon 15: 185-188.

Evans HC (2013) Fungal pathogens of spiders. In: Spider Ecophysiology (Nentwig W, ed.): 107-121. Berlin: Springer.

Evans HC, Samson RA (1982) Entomogenous fungi from the Galapagos Islands. Canadian Journal of Botany 60: 2325-2333.

Fries EM (1818) Observationes Mycologiae. Vol. 2 [Cancellans issue]. Copenhagen: $\mathrm{G}$. Bonnier.

Gams W, de Hoog GS, Samson RA, Evans HC (1984) The hyphomycete genus Engyodontium, a link between Verticillium and Aphanocladium. Persoonia 12: 135-147.

Gams W, Zare R (2001) A revision of Verticillium sect. Prostrata. III. Generic classification. Nova Hedwigia 72: 329-337.

Gams W, Hodge KT, Samson RA, Korf RP, Seifert KA (2005) Proposal to conserve the name Isaria (anamorphic fungi) with a conserved type. Taxon 54: 537.

Hodge KT, Gams W, Samson RA, Korf RP, Seifert KA (2005) Lectotypification and status of Isaria Pers.: Fr. Taxon 54: 485489.

Humber RA, Rocha LFN, Inglis PW, Kipnis A, Luz C (2013) Morphology and molecular taxonomy of Evlachovaea-like fungi, and the status of this unusual conidial genus. Fungal Biology 117: 1-12.

Humber RA, Rombach MC (1987) Torrubiella ratticaudata sp nov. (Pyrenomycetes: Clavicipitales) and other fungi from spiders on the Solomon Islands. Mycologia 79: 375-382.

Huang B, Li CR, Li ZG, Fan MZ, Li ZZ (2002) Molecular identification of the teleomorph of Beauveria bassiana. Mycotaxon 81: 229236.

Hywel-Jones NL, Samuels GJ (1998) Three species of Hypocrella with large stromata pathogenic on scale insects. Mycologia 90: 36-46.

Johnson D, Sung GH, Hywel-Jones NL, Luangsa-ard JJ, Bischoff JF, et al. (2009) Systematics and evolution of the genus Torrubiella (Hypocreales, Ascomycota). Mycological Research 113: 279-289.

Katoh K, Misawa K, Kuma K, Miyata T (2002) MAFFT: a novel method for rapid multiple sequence alignment based on fast Fourier transform. Nucleic Acids Research 30: 3059-3066.

Fig. 3. Representative taxa for Cordycipitaceae. A. Isaria sp. CEM 1729). B. Torrubiella arachnophila (rmk 12-001). C. Cordyceps bifusispora (CEM 1615). D. Cordyceps confragosa (CEM 1633). E. Isaria tenuipes (CEM 1032). F.Cordyceps militaris (CEM 740). G. Cordyceps rosea (CEM 1734). H. Cordyceps cf. cardinalis (CEM 1733). I. Cordyceps takaomontana with co-occurring I. tenuipes (CEM 1954). J. Cordyceps nelumboides (TNS 16306). Images not to scale. 
Katoh K, Toh H (2008) Recent developments in the MAFFT multiple sequence alignment program. Briefings in Bioinformatics 9: 286298.

Kepler RM, Sung G-H, Harada Y, Tanaka K, Tanaka E, et al. (2012) Host jumping onto close relatives and across kingdoms by Tyrannicordyceps (Clavicipitaceae) gen. nov. and Ustilaginoidea (Clavicipitaceae). American Journal of Botany 99: 552-561.

Kepler RM, Humber RA, Bischoff JF, Rehner SA (2014) Clarification of generic and species boundaries for Metarhizium and related fungi through multigene phylogenetics. Mycologia 106: 811-829.

Kobayasi Y (1941) The genus Cordyceps and its allies. Science Reports of the Tokyo Bunrika Daigaku, sect. B 5: 53-260.

Kobayasi Y, Shimizu D (1982) Cordyceps species from Japan 4. Bulletin of the National Science Museum Tokyo, ser. B 8: 79-91.

Kornerup A, Wanscher JH, Pavey D (1984) Methuen Handbook of Color. 3rd edn. New York: Hasting House.

Lebert H (1858) Ueber einige neue oder unvollkommen gekannte Krankheiten der Insekten, welche durch Entwicklung niederer Pflanzen im lebenden Körper enstehen. Zeitschrift für wissenschaftliche Zoologie 9: 439-453.

Li ZZ, Li CR, Huang B, Fan MZ (2001) Discovery and demonstration of the telemorph of Beauveria bassiana (Bals.) Vuill., an important entomogenous fungus. Chinese Science Bulletin 46: 751-753.

Link JH (1833) Handbuch zur Erkennung. Part 3. Berlin: S.J. Joseephy.

Linnaeus C (1753) Species Plantarum. 2 vols. Stockholm: L. Salvius.

Luangsa-ard JJ, Hywel-Jones NL, Samson RA (2004) The order level polyphyletic nature of Paecilomyces sensu lato as revealed through 18S-generated rRNA phylogeny. Mycologia 96: 773780 .

Luangsa-ard JJ, Hywel-Jones NL, Manoch L, Samson RA (2005) On the relationships of Paecilomyces sect. Isarioidea species. Mycological Research 109: 581-589.

Luangsa-ard JJ, Houbraken J, van Doorn T, Hong S-B, Borman AM, et al. (2011) Purpureocillium, a new genus for the medically important Paecilomyces lilacinus. FEMS Microbiology Letters 321: 141-149.

Mains EB (1949) New species of Torrubiella, Hirsutella and Gibellula. Mycologia 41: 303-310.

Mains EB (1950) Entomogenous species of Akanthomyces, Hymenostilbe, and Insecticola in North America. Mycologia 42: 566-589.

Mains EB (1958) North American entomogenous species of Cordyceps. Mycologia 50: 169-222.

McNeill J, Barrie FF, Buck WR, Demoulin V, Greuter W, et al. (eds) (2012) International Code of Nomenclature for algae, fungi, and plants (Melbourne Code). [Regnum vegetabile no. 154.] Königstein: Koeltz Scientific Books.

Nonaka K, Kaifuchi S, Ōmura S, Masuma R (2013) Five new Simplicillium species (Cordycipitaceae) from soils in Tokyo, Japan. Mycoscience 54: 42-53.

Petch T (1924) Studies in entomogenous fungus. IV. Some Ceylon Cordyceps. Transactions of the British Mycological Society 10: 28-45.

Petch T (1931) Notes on entomogenous fungi. Transactions of the British Mycological Society 16: 55-75.

Petch T (1934) Isaria. Transactions of the British Mycological Society 19: $34-38$.

Porter JR (1973) Agostino Bassi bicentennial (1773-1973). Bacteriological Reviews 37: 284-288.
Quandt CA, Kepler RM, Gams W, Araújo JPM, Ban S, et al. (2014) Phylogenetic-based nomenclatural proposals for Ophiocordycipitaceae (Hypocreales) with new combinations in Tolypocladium. IMA Fungus 5: 121-134.

Rehner SA, Buckley EP (2005) A Beauveria phylogeny inferred from ITS and EF1- $\alpha$ sequences: evidence for cryptic diversification and links to Cordyceps teleomorphs. Mycologia 97: 84-98.

Rehner SA, Minnis AM, Sung G-H, Luangsa-ard JJ, Devotto L, Humber R (2011) Phylogeny and systematics of the anamorphic, entomopathogenic genus Beauveria. Mycologia 103: 1055-1073.

Samson RA (1974) Paecilomyces and some allied hyphomycetes. Studies in Mycology 6: 1-119.

Samson RA, Evans HC (1974) Notes on entomogenous fungi from Ghana. II. The genus Akanthomyces. Acta Botanica Neerlandica 23: 28-35.

Sanjuan T, Tabima J, Restrepo S, Læssøe T, Spatafora JW, FrancoMolano AE (2014) Entomopathogens of Amazonian stick insects and locusts are members of the Beauveria species complex (Cordyceps sensu stricto). Mycologia 106: 260-275.

Sanjuan T, Franco-Molano AE, Kepler RM, Spatafora JW, Tabima J, et al. (2015) Five new species of entomopathogenic fungi from the Amazon in the genus Ophiocordyceps. Fungal Biology 119: 901-916.

Shimazu M, Mitsuhashi W, Hashimoto H (1988) Cordyceps brongniartii sp. nov., the teleomorph of Beauveria brongniartii. Transactions of the Mycological Society of Japan 29: 323-330.

Shrestha B, Hyun MW, Oh J, Han J-G, Lee TH, et al. (2014a) Molecular evidence of a teleomorph-anamorph connection between Cordyceps scarabaeicola and Beauveria sungii and its implication for the systematics of Cordyceps sensu stricto. Mycoscience 55: 231-239.

Shrestha B, Tanaka E, Han J-G, Oh J, Han SK, et al. (2014b) A brief chronicle of the genus Cordyceps Fr., the oldest valid genus in Cordycipitaceae (Hypocreales, Ascomycota). Mycobiology 42: 93-99.

Shrestha B, Tanaka E, Hyun MW, Han J-G, Chang SK, et al. (2016) Coleopteran and lepidopteran hosts of the entomopathogenic genus Cordyceps sensu lato. Journal of Mycology 2016, article ID 7648219 .

Spatafora JW, Quandt CA, Kepler RM, Sung G-H, Shrestha B, et al. (2015) New 1F1N species combinations in Ophiocordycipitaceae (Hypocreales). IMA Fungus 6: 357-362.

Sukarno N, Kurihara Y, llyas M, Mangunwardoyo W, Yuniarti E, et al. (2009) Lecanicillium and Verticillium species from Indonesia and Japan including three new species. Mycoscience 50: 369-379.

Sullivan RF, Bills GF, Hywel-Jones NL, White JF jr (2000) Hyperdermium: a new clavicipitalean genus for some tropical epibionts of dicotyledonous plants. Mycologia 92: 908-918.

Sung G-H, Spatafora JW, Zare R, Hodge KT, Gams W (2001) A revision of Verticillium sect. Prostrata. II. Generic delimitation using ribosomal DNA sequences. Nova Hedwigia 72: 311-328.

Sung G-H, Hywel-Jones NL, Sung J-M, Luangsa-ard JJ, Shrestha B, Spatafora JW (2007) Phylogenetic classification of Cordyceps and the clavicipitaceous fungi. Studies in Mycology 57: 5-59.

Talavera G, Castresana J (2007) Improvement of phylogenies after removing divergent and ambiguously aligned blocks from protein sequence alignments. Systematic Biology 56: 564-577.

Tsang C-C, Chan JFW, Pong W-M, Chen JHK, Ngan AHY, et al. (2016) Cutaneous hyalohyphomycosis due to Parengyodontium album gen. et comb. nov. Medical Mycology 54: 699-713. 
Vega FE, Goettel MS, Blackwell M, Chandler D, Jackson MA, et al. (2009) Fungal entomopathogens: new insights on their ecology. Fungal Ecology 2: 149-159.

Xiao G, Ying S-H, Zheng P, Wang Z-L, Zhang S, et al. (2012) Genomic perspectives on the evolution of fungal entomopathogenicity in Beauveria bassiana. Scientific Reports 2: 483.

Yarrow PJ [transl., Ainsworth GC, ed.] (1958) Bassi A [1835, 1836] Del Mal del Segno. [Phytopathological Classics no. 10.] Ithaca, NY: American Phytopathological Society Press.
Yip HY, Rath AC (1989) Microhilum oncoperae gen. et sp. nov. (Deuteromycotina: Hyphomycetes): the cause of mycosis on the larvae of Oncopera intricata (Lepidoptera: Hepialidae). Journal of Invertebrate Pathology. 53: 361-364.

Zare R, Gams W (2001) A revision of Verticillium section Prostrata. IV. The genus Lecanicillium and Simplicillium gen. nov. Nova Hedwigia 73: 1-50. 